\title{
Homozygous and compound heterozygous rare variants in VPS13C contribute to Lewy body diseases
}

\author{
Stefanie Smolders \\ VIN CMN - UAntwerp \\ Stéphanie Philtjens \\ VIB CMN-UAntwerp

\section{David Crosiers} \\ University of Antwerp

\section{Anne Sieben} \\ University of Ghent

\section{Elisabeth Hens} \\ VIB CMN-UAntwerp

\section{Bavo Heeman} \\ VIB CMN - UAntwerp \\ Sara Van Mossevelde \\ VIB CMN - UAntwerp \\ Philippe Pals \\ UAntwerpen
}

\section{Bob Asselbergh}

VIB CMN - UAntwerp

\section{Roberto Dos Santos Dias}

KU Leuven

\section{Yannick Vermeiren}

UAntwerp

Rik Vandenberghe

KU Leuven

\section{Sebastiaan Engelborghs}

UZ Brussels

\section{Peter P. De Deyn}

UAntwerpen

Jean-Jacques Martin

UAntwerpen

\section{Patrick Cras}

UAntwerpen

\section{Wim Annaert}

KU Leuven

Christine Van Broeckhoven ( $\sim$ christine.vanbroeckhoven@molgen.vib-ua.be )

VIB, University of Antwerp https://orcid.org/0000-0003-0183-7665 


\section{Research article}

Keywords: Lewy body diseases, Vacuolar Protein Sorting 13 homolog C, sporadic patients, recessive inheritance, loss-offunction, Oxford Nanopore Technologies long-read cDNA sequencing

Posted Date: May 22nd, 2020

DOI: https://doi.org/10.21203/rs.3.rs-29975/v1

License: (c) (i) This work is licensed under a Creative Commons Attribution 4.0 International License. Read Full License 


\section{Abstract \\ Background}

Dementia with Lewy bodies (DLB) and Parkinson's disease (PD) are clinically, pathologically and etiologically overlapping disorders. They are included in the Lewy body disease (LBD) continuum characterized by a-synucleinpositive Lewy body pathology in neurons. Homozygous PTC mutations in Vacuolar Protein Sorting 13 homolog $\mathrm{C}$ gene (VPS13C) are associated with early-onset PD.

\section{Methods}

Whole genome sequencing of two affected siblings and healthy parents of family $A$ with onset age below 50 years and DLB pathology confirmed at autopsy. Targeted resequencing of the VPS13C coding region in 844 LBD patients and 664 control individuals. Phasing cis-trans location of the compound heterozygous VPS13C variants. Analysis of VPS13C protein expression in lymphoblast cells and brain lysates. Immunohistochemistry and live cell labeling in HeLa and SHSY5Y cells overexpressing wild type or mutant VPS13C.

\section{Results}

In the two affected siblings of family A, we identified compound heterozygous rare variants located in trans in VPS13C Rare VPS13C variants (MAF $\leq 1 \%)$, are significantly associated $(p=0.0233)$ in the LBD patient cohort using optimized sequence kernel association test (SKAT-O). We identified 11 carriers of compound heterozygous variants and 1 carrier of homozygous variants in VPS13C. Trans location of the variants in compound heterozygous carriers was confirmed in 4 and cis location in 3 patients. The frequency of patient carriers with bi-allelic variants mimicking recessive inheritance is $1.07 \%$ (9/844). In post-mortem brain tissue of two unrelated DLB carriers of compound trans heterozygous variants, we observed a reduction of VPS13C protein expression. Overexpressing of wild type or mutant VPS13C, in HeLa and SHSY5Y cells, demonstrated that the mutations abolish the endosomal/lysosomal localization of VPS13C.

\section{Conclusions}

Our data indicate that rare alleles are associated with LBD, and when present bi-allelic in patients, these combinations have variable effects on expression and functioning of VPS13C. Apart from the recessive PTC mutations, some combinations of rare missense mutations might mimic recessive inheritance and explain part of the sporadic LBD patients. We propose that homozygous or compound heterozygous rare missense variants in VPS13C reducing VPS13C protein expression can contribute to risk of LBD.

\section{Background}

Lewy body diseases (LBD) are a heterogeneous group of neurodegenerative brain diseases characterized by the presence of Lewy bodies and Lewy neurites, mainly composed of aggregated a-synuclein' in neurons. Two disorders with substantial clinical, pathological and etiological overlap are at the two-side extremes of the LBD continuum: dementia with Lewy bodies (DLB) and Parkinson's disease (PD) [1, 2]. DLB is the second most common cause of neurodegenerative dementia [3-5] next to Alzheimer's disease (AD), with a worldwide prevalence of $0.37 \%$ in the 65 -plus group [6]. Clinical characteristics of DLB are fluctuating cognitive dysfunction, parkinsonism, visual hallucinations and delusions, and rapid eye movement (REM)-sleep behavior disorder [7]. Initial cognitive deterioration in DLB patients often 
resembles other dementias, but the presence of Lewy bodies, primarily in the brainstem, the limbic system or the neocortex differentiates, distinguishes DLB [8]. Also amyloid $\beta$ plaques, characteristic of AD pathology, are often observed together with Lewy bodies in DLB brains [2]. In the 65-min group, PD is second to AD with a prevalence of 2$3 \%$ [9]. Clinically, PD is primarily characterized by manifestation of parkinsonism with resting tremor, bradykinesia, rigidity and postural instability. Other non-motor symptoms may occur as well, including cognitive impairment, REMsleep behavior disorder, depression, constipation and autonomic dysfunction. Mild cognitive impairment and subsequent dementia is observed in $30 \%$ of PD patients [10]. PD patients have Lewy bodies restricted to the brainstem and the limbic system [11].

Of LBD patients, $85-95 \%$ are sporadic patients while families with a Mendelian segregating of disease were described [12-15]. Family-based and genome-wide association studies in PD cohorts identified over 70 loci with variable genetic contribution to PD risk $[15,16]$. Heterozygous mutations in SNCA, LRRK2 and VPS35 are a cause of autosomal dominant inherited PD, while homozygous and compound heterozygous mutations in PARK2, PINK1 and PARK7 are a cause of autosomal recessive inherited PD characterized by an early onset age. Moreover, $G B A$ is the most common genetic risk factor of PD with 7-12\% of PD patients carrying mono- or bi-allelic mutations in GBA and an estimated odds ratio of 5.4 $[18,24,74,75]$. More recently, new data showed that rare variants in VPS13C and ATP1OB, implicated in sporadic earlyonset PD patient are mimicking recessive inheritance [17-20]. PD patient carriers of pathogenic mutations in $S N C A$ or GBA have a higher risk of developing cognitive impairment and presenting a clinical phenotype of DLB [21-23]. So far, there is no causal gene for DLB identified, but genes implicated in AD or PD might have a potential role in the genetic etiology of DLB. Targeted approaches and genome-wide association studies (GWAS) associated SNCA and GBA loci and $A P O E 4$ allele status with increased risk of developing $\operatorname{DLB}[24,25]$. The knowledge acquired from the protein products of causal and risk genes provided valuable insights in disease mechanisms underlying $\operatorname{LBD}[3,17,33,44,78,117]$. Nonetheless causal genes in families and risk genes in patient cohorts, represent only a minor fraction of the genetic etiology of LBD. Here, we performed whole genome sequencing (WGS) of two siblings of healthy parents, with earlyonset (<50), pathologically confirmed DLB in the absences of mutation in known LBD genes to identify the underlying disease gene.

\section{Methods}

\section{Belgian patient and control cohorts}

Members of the Belgian BELNEU consortium recruited patients at neurological centers associated with university or general hospitals in Belgium [26, 27]. The LBD cohort comprised 844 LBD patients with mean age at onset age (AAO) of $62.9 \pm 11.8$ years (31.5\% female; $20.0 \%$ with positive familial history). In the LBD cohort, 233 patients were diagnosed with DLB (mean AAO $70.2 \pm 10.2$ years, range 34-88; 33.0\% females; $24.0 \%$ with positive familial history), of which 159 patients $(68.2 \%)$ had a clinical diagnosis and 74 patients (31.8\%) a neuropathological diagnosis of DLB. The remaining 611 patients in the LBD cohort had a clinical diagnosis of PD (mean AAO $60.6 \pm 11.3$ years, range 24-88; $31.1 \%$ females and $18.5 \%$ with positive familial history). Patients had a clinical examination by a neurologist and neuroimaging. We collected medical history of patients and family members. A positive family history of disease was given if at least one first-degree relative was affected. DLB patients were diagnosed in accordance with the established criteria for possible, probable or pathological DLB $[7,28]$, and PD patients according to the NINDS diagnostic criteria for PD [29]. The PD cohort was genetically profiled for the 5 major PD genes (SNCA, LRRK2, PARK2, PINK1 and PARK7) by means of Sanger sequencing for simple mutations and multiplex amplicon quantification (MAQ, Agilent, Multiplicom, Niel, Belgium), quantitative real-time PCR or multiplex ligation-dependent probe amplification (MLPA) [30] for copy number variants [31].

A geographically matched control cohort consisted of 664 individuals with a mean age at inclusion (AAI) of $72.0 \pm 9.4$ years (range 34-88) and comprised 60.5\% females. Control individuals were recruited among healthy partners of patients 
visiting a memory clinic, and negative for neurological or psychiatric antecedents or neurological complaints, or community-recruited individuals scoring $>25$ on a Montreal Cognitive Assessment (MoCA) [32] with a negative individual or familial history of neurodegenerative or psychiatric diseases.

\section{Ethical assurances}

The ethics committee of the University Hospital Antwerp and University of Antwerp approved the clinical and genetic research protocols. Collection of biological samples was in accordance with the written informed consent signed by the participant and/or their legal guardian.

\section{Whole genome sequencing}

Short-read paired-end WGS of two affected siblings of family A (Fig. 1a), subsequent read alignment to the human reference genome (GRCh37/hg19) and base and variant calling were performed by Complete Genomics ${ }^{\mathrm{TM}}$ Inc [33]. To annotate and select genetic variants in the WGS data we used GenomeComb [34]. High quality variants were selected based on a sequence coverage of at least $20 \mathrm{x}$, a variant call score (Complete Genomics Inc.) of $\geq 60 \mathrm{~dB}$, i.e. a probability of $\geq 10^{6}: 1$ describing the likelihood of the variant call compared to the second most likely call, and genomic location outside repeat regions marked as simple repeats or micro satellites by RepeatMasker v3.0 [35]. Novel or rare (minor allele frequency $(\mathrm{MAF})<1 \%$ ) variants in the 1000 Genome Project database [36] and below 25\% in our in-house next generation sequencing database of Belgian patients with distinct neurological disorders), non-synonymous coding and splice site variants were selected. Finally, we focused on variants in line with autosomal recessive inheritance, i.e. homozygous or compound heterozygous variants. We slected homozygous variants and multiple compound heterozygous variants in the same gene.

\section{Targeted resequencing of VPS13C}

We performed PCR amplification of all 86 coding exons and flanking splice sites of VPS13C by amplicon-target PCR amplification (MASTR technology; Agilent). Amplicons were uniquely tagged; based on the Nextera XT shotgun library preparation protocol (Illumina), containing sample-specific indices [37]. Libraries $(n=384)$ were pooled and sequenced in one run on the MiSeq platform using the MiSeq V3 chemistry, generating paired-end sequence reads of 300 nucleotides (Illumina). After sample de-multiplexing, we mapped sequence reads using the Burrows-Wheeler Aligner (BWA) [38, 39] to a mini-genome, combining the target sequences extracted from the human genome, reference sequence hg19. Sequence variants were called using GATKv3.5 HaplotypeCaller [40,41] and variants annotated using GenomeComb [34]. We used the Combined Annotation Dependent Depletion (CADD_Phred) score, to predict the deleterious impact of nonsynonymous variants in VPS13C, whereby a score above 20 represents the top $1 \%$ most deleterious variants in the genome [42]. Alternative splicing of VPS13C produces four different transcripts, ubiquitously expressed. Coding variants are numbered relative to the translation initiation codon in the largest VPS13C transcript (GenBank Accession Number NM_020821.2. Amino acid changes, numbered according to the largest VPS13C isoform (GenPept Accession Number NP_065872.1). Sequencing reads, visualized with the Integrative Genomics Viewer (IGV) [43] using BAM files of individual samples. 


\section{Sanger sequencing}

VPS13C exons 7-8, 27, 37-38, 41, 46, 54, 60-61, 70-73, 76-77 and 80, <85\% 20X covered with the MASTR assay, were analyzed with Sanger sequencing and rare $(\mathrm{MAF}<1 \%)$ non-synonymous coding and splice site variants, identified by targeted resequencing, were validated and genotyped in available family members with Sanger sequencing. Primers were designed using the online Primer3 software [44]. Target regions were PCR amplified from genomic DNA and subsequently Sanger sequencing using the BigDye ${ }^{\circledR}$ Terminator Cycle Sequencing kit v3.1 (Applied Biosystems) on an ABI3730 automated sequencer (Applied Biosystems). Sanger sequences were analyzed using Seqman (DNASTAR) and NovoSNP software [45].

\section{Allele-specific PCR to determine allele-phase configuration}

We used allele-specific PCR amplification to determine cis/trans configuration of two VPS13C variants present in the same exon. For each mutation, an allele-specific and a wild-type primer were designed in combination with a general second primer using the online Primer3 software [44] to amplify both the wild-type and mutant allele separately (Table S8, ). The amplicon containing the location of the second mutation purified and Sanger sequenced (as described above).

\section{Haplotype sharing analysis}

For haplotype sharing, we selected 11 polymorphic STR markers surrounding VPS13C at chromosome 15q21 for genotyping: D15S1008, D15S198, D15S155, chr15:62080540-62080584, chr15:62440295-62440338, D15S1036, D15S997, D15S159, D15S993 and D15S1507. The STR markers were PCR amplified using fluorescently labelled primers and size-separated using GeneScan 500 Liz Size Standard (Applied Biosystems) on an ABI3730xI DNA Analyzer (Applied Biosystems). Local Genotype Viewer, used to score fragment lengths.

\section{In silico prediction of splice-site variants}

For evaluation of the of splice site variants in silico we used five splicing prediction programs (SpliceSiteFinder-like, MaxEntScan, NNSPLICE and GeneSplicer) integrated in Alamut Visual version 2.11.0 (Interactive Biosoftware).

\section{Cell culture}

Lymphoblast cells, immortalized by Epstein Barr virus transformation of lymphocytes collected from whole blood on lithium heparin according to standard procedures [46, 47], were cultured in Roswell Park Memorial Institute 1640 medium (RPMI 1640; Life Technologies), supplemented with 15\% fetal calf serum (Sigma Aldrich), 2 mM L-glutamine (Life Technologies) and $500 \mathrm{U} / 500 \mu \mathrm{g}$ penicillin/streptomycin (Life Technologies).

Human cervical carcinoma (HeLa) cells were cultured in Modified Eagles medium (MEM; Life Technologies), supplemented with $10 \%$ fetal calf serum (Sigma Aldrich) and $500 \mathrm{U} / 500 \mu \mathrm{g}$ penicillin/streptomycin (Life Technologies). Human neuroblastoma cells (SH-SY5Y) were cultured in Modified Eagles medium (MEM; Life Technologies) 
supplemented with 10\% fetal calf serum (Sigma Aldrich), 1\% non-essential amino acids (Life Technologies), $2 \mathrm{mM}$ glutamine (Life Technologies) and $500 \mathrm{U} / 500 \mu \mathrm{g}$ penicillin/streptomycin (Life Technologies).

\section{Long-read cDNA sequencing}

Total RNA was isolated and purified from lymphoblast cells using the RNeasy Mini Kit as recommended by the manufacturer (Qiagen), and subsequently treated with DNase (Turbo DNase Kit; Ambion). Polyadenylated RNA was enriched from total RNA samples using the Poly(A) RNA Selection Kit (Lexogen; M039100). First strand synthesis, performed on 50ng Poly A+ RNA by means of the SuperScript IV First-Strand Synthesis System using VN primer (VNP), which anneals to poly A-tails, and Template Switching Oligonucleotides (TSO) (Table S8). Both oligonucleotides have sequence tags for subsequent second strand generation and PCR amplification using a Forward and Reverse primer (Table S8). cDNA amplification of VPS13C fragments containing the double variants was performed using LongAmp $B$ Taq DNA Polymerase (New England Biolabs) using primers designed with the online Primer3 software (Table S8, ) [44]. Native barcoding and adapter ligation were carried out using the Native Barcoding Expansion 1-12 (EXP-NBD104; Oxford Nanopore Technologies, ONT) in conjunction with the Ligation Sequencing Kit (SQK-LSK109; ONT).

VPS13C amplicons from 11 individual samples were pooled equimolar and sequenced on a MinION, using a single FLOMIN106 flow cell (ONT). In total $900 \mathrm{Mb}$ of data was generated ( $650 \mathrm{~K}$ reads). Base calling and barcode de-multiplexing of the raw data was performed with Guppy (v.3.2.2). Further analysis was performed with a pipeline integrated in GenomeComb [34]. Alignment to the hg38 reference sequence [48] was performed with minimap2 using the splicedhq preset [49]. Samtools was used for the conversion to BAM and sorting [50], which enabled visualization with IGV [43]. Single nucleotide variant calling and haplotyping was performed using longshot (v0.4.0) [51].

\section{Splice-site variant analysis on cDNA}

Lymphoblast cells were treated with $100 \mu \mathrm{g} / \mathrm{ml}$ cycloheximide (Sigma-Aldrich BVBA) or equal amounts of dimethyl sulfoxide (Fisher Scientific) for $4 \mathrm{~h}$. The RNeasy procedure (RNeasy Mini Kit; Qiagen) was used to isolate and purify total RNA from lymphoblast cells as recommended by the manufacturer. Subsequently, total RNA was treated with DNase (Turbo DNase Kit; Ambion). cDNA synthesis of total RNA was performed primed with random hexamer primers using the SuperScript® III First-Strand Synthesis System for RT-PCR (Invitrogen), followed by a RNase H digestion to remove the RNA template from the cDNA:RNA hybrid molecule. To investigate the effect of VPS13C c.4166-8C>A on splicing, primers were designed using the online Primer3 software (Table S8, ) [44]. Exon skipping of exon 38 due to VPS13C c.4166-8C>A was investigated with a F primer located in exon 37 and a R primer located in exon 39. VPS13C cDNA was amplified in a total volume of $15 \mu \mathrm{l}$. Fragments were analyzed on a $2 \%$ agarose gel, and sized using Tracklt ${ }^{\text {TM }} 100$ bp DNA Ladder (Invitrogen). Additionally, the VPS13C amplicons were purified and Sanger sequenced (as described above).

\section{Quantitative RT-PCR}

Total RNA was isolated from lymphoblast cells, using the Ribopure kit (Ambion) or the Magtration Reagent MagDEA RNA 100 kit (Precision System Science), and was treated with DNase (Turbo DNase Kit; Ambion). First-strand cDNA was synthesized with the SuperScript III First-Strand Synthesis System (Invitrogen) utilizing random hexamer and oligo dT primers. PCR was carried out for 40 cycles with a VPS13C-specific forward and reverse primer (Table S8), using the Power SYBR Green PCR Master Mix (Life Technologies) on an ABI Viia ${ }^{\text {TM } 7 ~ R e a l-T i m e ~ P C R ~ S y s t e m ~(A p p l i e d ~ B i o s y s t e m s) . ~}$ 
Relative expression levels were quantified against two housekeeping genes, TBP and $h Y W A Z$, using qbase software (Biogazelle, Ghent, Belgium).

\section{Generation of VPS13C antibody}

The cDNA encoding the amino acids 3621-3742 of human VPS13C, isoform 2A (NM_020821), was PCR amplified, using primers carrying the restriction sites for Nhel and Xhol (Table S8, ), and subsequently cloned into a pET28a vector (Novagen), in frame with an N-terminal 6xHis tag (pET28-6xHis-VPS13C.2). The construct was transformed into BL21(DE3) competent E. coli (New England Biolabs) and expressed for $3 \mathrm{~h}$, at $28^{\circ} \mathrm{C}$, to allow proper folding of the protein. Expressed recombinant protein was purified with Ni-NTA resin (Qiagen) followed by gel filtration chromatography using an $\ddot{\text { AKTA }}{ }^{\mathrm{TM}}$ pure system equipped with a HiLoad 26/600 Superdex 75 pg column (GE Healthcare). Purity of recombinant protein was assessed by Coomassie Brilliant blue staining. New Zealand white rabbits were immunized with different amounts of purified recombinant protein, and the serum was subsequently tested by Western blot for VPS13C immunoreactivity, using VPS13C knockout HeLa cell extracts as a negative control (Fig. S12). Polyclonal antibodies from the sera displaying the highest titer were affinity purified against the recombinant protein immobilized on NHSactivated Sepharose ${ }^{\circledR} 4$ Fast Flow (GE Healthcare) according to the manufacturer's instructions. Antibodies were eluted from the column with $100 \mathrm{mM}$ Glycine $\mathrm{pH} 2.7$ and immediately neutralized with $1 \mathrm{M} \mathrm{Tris-} \mathrm{HCl} \mathrm{pH} \mathrm{9.0.} \mathrm{The} \mathrm{eluted} \mathrm{fraction}$ was dialyzed against PBS for 48h, with three daily buffer changes. Finally, purified antibodies were concentrated to 1 $\mathrm{mg} / \mathrm{mL}$ using a Vivaspin 15R, 30000 MWCO hydrosart (Sartorius AG), and frozen in aliquots containing 50\% glycerol.

\section{Western blotting}

Protein analysis performed in lymphoblast cells and brain lysates of patient carriers of $>1$ VPS13C variants and of family A. Cells and brain tissue were lysed in modified radioimmune precipitation buffer (RIPA: 1\% sodium dodecyl sulfate, 150 $\mathrm{mM} \mathrm{NaCl}, 0.5 \% \mathrm{Na}-\mathrm{Doc}, 1 \% \mathrm{NP}-40,50 \mathrm{mM}$ Tris-HCL; $\mathrm{pH}, 8.0)$ supplemented with protease and phosphatase inhibitor (2x Complete Protease inhibitor cocktail and 1x Phosphostop phosphatase inhibitor cocktail; both from Roche). Lysates were sonicated on ice, cleared at 20,000 g for $15 \mathrm{~min}$ at $4^{\circ} \mathrm{C}$, and supernatants were collected for immunoblotting. Protein concentrations were measured using a BCA Protein Assay Kit (Pierce ${ }^{\mathrm{TM}}$; Thermo Fisher Scientific). Equal amounts of proteins were separated on a 3-8\% Tris-Acetate gel (Life Technologies) and electro-blotted onto a polyvinylidene difluoride membrane (Hybond P; Amersham Biosciences). Membranes were blocked in 5\% skimmed milk in PBS and probed with primary antibodies against VPS13C (polyclonal rabbit anti-human VPS13C (1:1,000; Novus Biologicals; NBP1-94043), polyclonal rabbit anti-human VPS13C (1:1,000; Novus Biologicals; NBP1-94044) or polyclonal rabbit antihuman (1:2,000; generated in house as described above)) and GAPDH (monoclonal mouse anti-human GAPDH (1:20,000; Genetex; GTX627408)). Immunodetection was performed with specific secondary antibodies conjugated to horse-radish peroxidase in combination with the ECL prime chemiluminescent detection system (GE Healthcare) or the WesternBright Sirius detection system (Isogen Life Science). The band intensities were determined by quantifying the mean pixel grey values in a rectangular region of interest using Image Quant TL software (GE Healthcare Life Sciences) and subsequently normalized to GAPDH.

\section{Preparation of cDNA constructs}

The codon optimized coding sequence of wild type (WT) human VPS13C was purchased in a Gateway ${ }^{\circledR}$ compatible pDONR vector from GeneArt ${ }^{\mathrm{TM}}$ Gene synthesis (pDONR-VPS13C ${ }^{\mathrm{WT}}$; Life Technologies). The VPS13C missense variants 
p.Trp395Cys and p.Ala444Pro were introduced into the pDONR-VPS13C ${ }^{\text {WT }}$ vector with QuickChange Site-Directed Mutagenesis. Sequence verified mutant entry clones were subcloned into the Gateway® ${ }^{\circledR}$ pcDNA ${ }^{\mathrm{TM}}$-DEST40 Vector with a C-terminal V5-6x His tag (Thermo Fisher Scientific) or into an in-house developed Gateway®-compatible pCR3 Vector with a C-terminal EmGFP-tag. Sequences were verified by DNA sequencing and primers used for cloning are listed in Table S8 ().

\section{Transfections}

HeLa or SH-SY5Y cells were transfected using either X-tremeGENE 9 DNA Transfection Reagents (Sigma Aldrich), Lipofectamine LTX plus (Invitrogen), both according to manufacturer's protocol, or Polyethylenimine (PEI) according to an in house optimized protocol. Briefly, cells were seeded in a 6-well plate at $2.5 \times 10^{5}$ cells per well, 24 hours before transfection. On the day of transfection, cell medium was replaced by medium without antibiotics. 1.44 $\mu$ g plasmid DNA was diluted in $115 \mu \mathrm{l}$ Opti-MEM (Life-Technologies) and in parallel, $7.21 \mu \mathrm{l} \mathrm{PEI} \mathrm{was} \mathrm{diluted} \mathrm{115 \mu l} \mathrm{Opti-MEM.} \mathrm{The} \mathrm{diluted}$ PEI was added to the DNA and mixed gently by vortexing. After $10 \mathrm{~min}$ of incubation at room temperature, the solution was added to the cells. All cells were evaluated $48 \mathrm{~h}$ post-transfection.

\section{Immunohistochemistry and live cell labeling}

HeLa or SH-SY5Y cells were grown on $12 \mathrm{~mm}$ glass coverslips (Fisher Scientific), fixed for 20 min with $4 \%$ paraformaldehyde (PFA; Laborimpex) in PBS at room temperature and washed three times in PBS. The cells were permeabilized in $0.25 \%$ Triton X-100 for 10 min, washed three times in PBS and blocked for $1 \mathrm{~h}$ in $5 \%$ BSA (Merck) with normal donkey serum (1:500; Merck). Cells were then incubated overnight at $4^{\circ} \mathrm{C}$ with one or a combination of the following antibodies: monoclonal mouse anti-V5 tag (1:400; Life Technologies; R960-25), polyclonal goat anti-V5 tag (1:2,000; Abcam; ab9137), polyclonal rabbit anti-human Giantin (Golgi; 1:10,000; Covance; 924302), polyclonal rabbit anti-human TGN46 (Trans-Golgi Network; 1:800; Abcam; ab50595), monoclonal mouse anti-human PDI (endoplasmic reticulum (ER); 1:100; Abcam; ab2792), monoclonal mouse anti-human LAMP1 (lysosomal-associated membrane protein 1; 1:200; Developmental Studies Hybridoma Bank; H4A3), polyclonal rabbit anti-human Rab7 (late endosomes; 1:200; Sigma Aldrich; R4779) and monoclonal mouse anti-human CD63 (early endosomes; 1:250; Abcam, ab8219). To visualize the primary antibodies, the cells were incubated with secondary antibodies conjugated to Alexa Fluor 488, Alexa Fluor 594 or Alexa Fluor 647 (all 1:500; Thermo Fisher/Life Technologies) for $1 \mathrm{~h}$ at room temperature.

For live cell imaging, transfected HeLa cells were plated on a standard $35 \mathrm{~mm}$ glass bottom dish (MatTek Corporation, P35G-1.5-14-C) and incubated for $30 \mathrm{~min}$ at $37^{\circ} \mathrm{C}$ with growth medium containing 50nM LysoTracker ${ }^{\circledR}$ probe (Thermo Fisher). After incubation, the medium was replaced by FluoroBrite DMEM (Life Technologies).

\section{Image acquisition and analysis}

Images were taken with a Zeiss LSM700 confocal microscope using either a 63x/1.40 Plan-Apochromat or a 40x/1.30 Plan-Neofluar objective. Filters, dichroics and scanning modes were set to exclude crosstalk between the different fluorescence channels, pixel sizes were set according to the Nyquist sampling theorem and z-stacks comprising entire cells, acquired at optimal step sizes. To visualize co-localization of VPS13C with different organelle markers, Fiji software generated line-intensity plot profiles [52]. For quantification of VPS13C mislocalization, two researchers who were blind for the genotype visually scored cells. 


\section{Neuropathology}

Brains of patients $\mathrm{P} 1$ and $\mathrm{P} 3$, fixed in formalin for 13 weeks and 5 weeks, respectively. Samples, taken from the frontal cortex areas $4,6,8,9,10,11,12,24$ and 46 , the superior temporal cortex, hippocampus and amygdala, parietal and occipital cortex, the thalamus, neostriatum, pallidum, mesencephalon, pons, medulla oblongata and cerebellum. Cytological stains included Cresyl-Violet, Hematoxylin-Eosin, and Klüver-Barrera as myelin stain. Immunohistochemistry, performed with antibodies against $\beta$-amyloid (4G8), hyperphosphorylated tau (AT8), ubiquitin, TDP-43, FUS, p62 and asynuclein.

\section{Statistics}

Burden and variance-component tests implemented in the optimized sequence kernel association test (SKAT-0) provided in the R package SKAT v2.0.0, used to investigate association of single VPS13C variants with MAF $\leq 1 \%$ and PD. First, power calculation was performed within the SKAT framework using a logistic test for dichotomous traits (target sequence: $12,941 \mathrm{bp}$, causal variant percentage $=20 \%$, negative variant percentage $=20 \%$, Maximal OR $=5$ ). Under these conditions, the total sample cohort required to reach $80 \%$ power with a 0.05 significance level is at least 1050 individuals. Our patient and control cohort consisted of 1508 individuals and met the requirements. Adjustment to SKAT$O$ applied taken the small sample size ( $<2000$ individuals). Gender was included as covariate. We considered a twosided $p$ value $<0.05$ significant. To investigate association between bi-allelic VPS13C mutations and PD, we compared statistically the mutation frequencies between the patient and control group using Fisher's exact statistics, and we considered a $p$-value of $<0.05$ significant. Data are represented as the average \pm standard deviation of a minimum of 3 independent experiments. For the description of the statistical significance of differences, the Multiple Comparisons of a one-way ANOVA using the GraphPad Prism V7.01 software calculated P-values. Values were considered significant if * $0.01<P<0.05 ; * \star 0.001<P<0.01 ; * \star \star 0.0001<P<0.001 ; * \star \star \star P<0.0001$.

\section{Results}

\section{Identification of compound heterozygous VPS13C missense variants in family A}

In family A (Fig. 1a), genomic DNA was available of two affected siblings and their unaffected parents. The index patient, P1 (II.2. Fig. 1a), first presented with non-fluent aphasia at age 42. A clinical neurological examination revealed non-fluent aphasia, extrapyramidal signs consisting of hypomania, bradykinesia, gait disturbances, cogwheel rigidity and resting tremor (Table S1). Frontal disinhibition signs such as glabella reflex, snout reflex and palmomental reflexes were present. Later in the disease course, myoclonus was present and behavioral symptoms became more apparent. The disease progressed rapidly and the patient died at the age of 54 years. Patient P1 (II.2, Fig. 1a) received a

neuropathological confirmation of diffuse LBD, neocortical type. The affected sibling of patient P1 (II.1, Fig. 1a), presented at age 41 years with initial symptoms of anxiety and depression, combined with word finding difficulties and an episode of visual hallucinations and delusion. The patient received a clinical diagnosis of unspecified dementia and died at the age of 47 years. There were no other familial antecedents of early-onset neurodegenerative brain diseases. Both parents were unaffected at advanced ages (> 80 years), showing that segregation of the disease in family $A$ is consistent with autosomal recessive inheritance (Fig.1a).

We obtained WGS data of the two affected siblings of family A (Fig. 1a). Variant filtering resulted in 178 rare, nonsynonymous coding and splice site variants (MAF < 1\%) shared by the two affected siblings. In four genes, Cellular 
Communication Network Factor 6 gene (CCN6), Ring Finger Protein 6 gene (RNF6), Chronic Lymphocytic Leukemia Upregulated 1 gene (CLLU1) and the Vacuolar Protein Sorting 13 homolog $C$ gene (VPS13C), we observed several putative compound heterozygous variants shared by the affected siblings but no homozygous variants (Table S2, ). Genotyping of all putative compound heterozygous variants in the unaffected parents showed that only the VPS13C missense variants, p.Trp395Cys/p.Ala444Pro, were compound heterozygous (trans configuration) with each parent carrying one allele (Fig. 1a; Table 1). The multiple heterozygous variants in WISP3, RNF6 and CLLU1 were all in cis configuration in one parent and absent in the other parent. The VPS13C p.Trp395Cys and p.Ala444Pro alleles were absent in the control cohort of 664 individuals and belong to the $1 \%$ most deleterious substitutions in the human genome, indicated by a CADD_Phred score above 20 (Table 1) [42].

\section{Additional VPS13C rare variants in the LBD families and patient cohort}

Targeted resequencing of the 86 coding exons and splice site junctions of VPS13C in the LBD patient and control cohorts identified 71 non-synonymous coding and splice site rare variants with a MAF $\leq 1 \%$ in VPS13C (Table S3, Fig. S1). All VPS13C variants were present in isoform 2, the largest VPS13C transcript (NM_020821.2), containing exons 6 and 7 , and the main splice variant in brain, suggesting brain-specific gene functions [53]. To study a potential role of these rare non-synonymous or splice VPS13C variants in LBD, we performed a SKAT-O analysis on the variants listed in Table S3. In the Belgian cohort, 86 LBD patients (86/844, 10.2\%), including 22 DLB (22/233, 9.44\%) and 64 PD (64/611, $10.47 \%$ ) patients, carried a rare variant in VPS13C, compared to 86 control individuals $(13.0 \%)$. We observed a significant association $(p=0.233)$ between rare variants and LBD.

We identified one patient, P2, clinically diagnosed with DLB and homozygous for VPS13C p.Ala444Pro, located on different haplotypes of which one shared with the heterozygous p.Ala444Pro carriers in family A (Table 1, Fig. S2c). Further, we observed 10 patients and 7 controls with rare compound heterozygous variants in VPS13C (Table 1, Table S5)

We phased the compound heterozygous VPS13C variants to identify carriers with trans configuration in line with recessive inheritance. Besides patient P1 (Family A, Fig. 1a), we identified 3 additional patients, P3, P4 and P5, carrying trans compound heterozygous variants in VPS13C (Table 1). Genotyping the two VPS13C variants and haplotype sharing analysis in relatives revealed that the child of patient P3 (Family B, Fig. 1a) carried one allele, p.Thr1218Ala, confirming that the compound p.Thr1218Ala/p.lle2789Thr variants are in trans configuration in the affected parent (Fig. 1a. Fig. S2b). In the P4 patient, the compound p.Met2711/le/p.Ile2789Thr variants are located in exon 61 and allelespecific PCR analysis confirmed trans configuration (Fig. S3). In the P5 patient, long-read cDNA sequencing demonstrated trans configuration for p.Ala1687Val/p.Ser2904Leu and different haplotypes. In addition, we identified 3 patients and 5 controls with compound heterozygous variants in cis configuration (Table S5, Table S6). Of the patient P10 in Family C (Fig. 1a), the two children were negative for both VPS13C variants, p.Ser963Gly/p.Ser2026Phe, present in the compound heterozygous parent. The p.Lys171Glu/c.4166-8C>A variants are present in patient $\mathrm{P} 11$ and three controls C3, C4 and C5. The relative high frequency in the Belgian cohorts of this VPS13C variant combination (4/1506; $0.27 \%$ observed; $0.00027 \%$ expected) (Table S5, Table S6), we suspected that the two variants were located in cis in all four carriers. We were able to confirm the cis configuration by haplotype sharing analysis showing a common haplotype shared by all carriers (Fig. S2c). In the control C6, the VPS13C p.Met2764lle/p.Val2765Leu variants are located in cis based on their joint occurrence in sequencing reads obtained via targeted resequencing of VPS13C (Fig. S4). Long-read cDNA sequencing in patient P12 and control C7 confirmed a shared haplotype and cis configuration for p.Ser963Gly/p.Ser2026Phe (P12) and p.Ser2282Phe/p.Arg3176Gly (C7).

Page $11 / 27$ 
To summarize, we identified 1 patient with homozygous and 11 patients with compound heterozygous rare variants in VPS13C. Four patients have a confirmed trans and 3 patients confirmed cis configuration of the compound variants in VPS13C. The 4 remaining patients have an unknown variant configuration. The frequency of patient carriers with biallelic variants, including the 4 non-phased patients is 1.07\% (9/844) (Table 1). All their compound mutated alleles are clustering in VPS13a involved in lipid transport, the putative WD40 involved in late endosomal/lysosomal localization or the pleckstrin homology involved in lipid droplet binding, domain of VPS13C (Fig. 1b) [54]. The clinical characteristics of all 9 patient carriers are summarized in Table S1. All were negative for mutations in the major PD genes and other genes associated with neurodegenerative brain diseases (Table S4), except for the PD patient P9, with the VPS13C p.lle2789Thr/p.lle3726Val un-phased alleles, who also carries the LRRK2 p.R1441C mutation. Of the 7 compound heterozygous carriers in the control group, 5 controls have a confirmed cis configuration of their VPS13C variants while the 2 remaining controls had no confirmed phasing $(2 / 664 ; 0.30 \%)$.

\section{Effect of VPS13C splice site variants on mRNA splicing.}

Apart from the missense variants we also identified three splice site variants, $c .448+7 A>G, c .4056+3 A>C$ and $c .4166-$ $8 \mathrm{C}>\mathrm{A}$, each partnering with a missense mutation, in control $\mathrm{C} 2$, patient $\mathrm{P} 8$ and in patient $\mathrm{P} 11$ and controls $\mathrm{C} 3, \mathrm{C} 4$, and $\mathrm{C} 5$ (Table1, Table S5, Table S6). The c.4056+3A>C variant in the PD patient P8 was absent in 664 controls and predicted to affect the canonical splice donor site of exon 36 by three in silico splicing prediction programs (Table S7). Of patient P8 we had no lymphoblast cells or brain to evaluate the actual effect on mRNA splicing. The splicing predictions for c.4166$8 C>A$ and $c .448+7 A>G$ were inconsistent between prediction programs (Table S7). We did not observed exon skipping of c. $4166-8 C>A$ when treating the lymphoblast cells of the 4 carriers with cycloheximide before isolating total mRNA (Fig. S5).

\section{Reduced expression for VPS13C mutant alleles}

To determine the effect VPS13C missense variants on transcript and protein expression we used qPCR and Western blot analysis of lymphoblast cells of DLB patient P1 (Family A, Fig 1a, II.2) and unaffected parents (Fig. 2a-C). No lymphoblast cells were available of the affected sib of P1 (Fig. 1a, II.1). We did not observe significant difference in VPS13C transcript expression for patient P1 and parents, compared to controls negative for rare VPS13C variants (Fig. $2 a)$. However, endogenous VPS13C protein expression were reduced $\sim 40 \%$ in the parent carrying the p.Ala444Pro allele $(p=0.0618)$ and $\sim 70 \%$ in the parent carrying the $p$.Trp395Cys allele $(p=0.0024)$. In the patient P1, carrying both VPS13C alleles p.Trp395Cys/p.Ala444Pro there is $90 \%$ reduction $(p=0.0002)$, compared to the control individuals (Fig. $2 b$ and 2c). We also investigated the protein expression in all patient and control carriers of compound heterozygous VPS13C variants and lymphoblast cells available. We observed a reduction in VPS13C expression in lymphoblast cells of patient P2, homozygous for p.Ala444Pro, patient P5 trans compound heterozygous for p.Ala1687Val/p.Ser2904Leu and patient P7 un-phased compound heterozygous for p.Thr766Ala/p.Leu1846Ser, compared to control individuals (Fig. 2d, e). None of the control carriers showed a reduction in VPS13C protein expression (Fig. 2d, e). We observed the largest reductions in patients $\mathrm{P} 1$ and $\mathrm{P} 2$, indicating that the $\mathrm{p}$.Trp395Cys and p.Ala444Pro mutated alleles had the strongest effect compared to the other VPS13C alleles in lymphoblast cells (Fig. 2d). Brain tissue was available of patient carriers P1 and $\mathrm{P} 3$, and two unrelated control individuals, negative for rare VPS13C variants (MAF $\leq 1 \%$ ). In all studied brain tissues and brain regions (prefrontal cortex, temporal cortex, cerebellar cortex, hippocampus, substantia nigra and nucleus caudatus), VPS13C protein expression was abnormally reduced in patients compared to control individuals, with almost no VPS13C protein levels in patient P1 (Fig. 2f) of Family A (Fig. 1a), the discovery family we used for gene identification. 


\section{Subcellular localization of VPS13C}

We transfected HeLa cells with wild type or mutant VPS13C variants (p.Trp395Cys or p.Ala444Pro) and investigated the effect of the missense variants on the subcellular localization of the protein. Wild type VPS13C localized to small organelles, whereas mutant VPS13C alleles, p.Trp395Cys or p.Ala444Pro, localized at larger cytosolic structures in the majority of cells (Fig. 3a-b). Triple immunostaining of V5 (VPS13C constructs with a C-terminal V5-6x His tag), with markers for late endosomes (Rab7) and lysosomes (Lamp1), revealed a late endosomal/lysosomal localization of wild type VPS13C. However, the late endosomal/lysosomal localization of VPS13C was lost when the VPS13C mutant alleles were present (Fig. 3C), which was independently confirmed with GFP-tagged VPS13C constructs and other markers (CD63, late endosomes; Lysotracker, lysosome; Fig. S6, S7). Moreover, we could confirm this subcellular localization of wild type and mislocalization of mutant VPS13C at late endosomes and lysosomes in human neuroblastoma SH-SY5Y cells (Fig. S8). Immunostaining in HeLa cells with markers for the ER (PDI; Fig. S9), cis- and medial-Golgi (Giantin; Fig. S10) and trans-Golgi (TGN46; Fig. S11) demonstrated no co-localization of wild type and mutant VPS13C with these organelles.

\section{Pathological phenotype of compound heterozygous missense mutation carriers}

Patient P1 died at age 54, and we obtained and autopsy with 12 hours post mortem delay (PMD). We observed moderate frontotemporal atrophy with the superior temporal gyrus affected more than the medial temporal gyrus (Fig. 4a). In the midbrain, the zona compacta of the substantia nigra was very pale. The substantia nigra showed severe neuronal loss, most explicit in the lateral part of the zona compacta. Using the rating scheme for cerebrovascular lesions of Deramecourt and colleagues, no more than grade 2 of vascular pathological alterations could be scored [55]. The lateral occipitotemporal gyrus of patient P1 showed microspongiotic changes in the cortex (Fig 4c). The hippocampus and parahippocampal gyrus were affected with a moderate number of neurofibrillary tangles, neuritic threads and dystrophic neurites (Fig. 4d), whereas other brain structures did not present tau pathology. 4G8 staining to detect $\beta$-amyloid pathology and TDP-43 and FUS staining for frontotemporal dementia (FTD) pathology were all negative. The a-synuclein staining showed an abundance (grade 3) of Lewy bodies and Lewy neurites in the frontal cortices, temporal neocortex, hippocampus, parahippocampal gyrus, amygdala, and in the pigmented nuclei of the mesencephalon, pons and medulla oblongata (Fig. 4e, i-k). Rare Lewy body pathology was found in the occipital cortex, neostriatum, hypothalamus. Based on our neuropathological findings, the patient received a diagnosis of diffuse Lewy body disease, neocortical type [7,56].

Patient P3 died at age 64 and autopsy brain was obtained 8 hours following death. We observed severe atrophy of the temporal lobe while the parietal and occipital lobes were less severely affected (Fig. 4b). Ventricular dilation was severe and most explicit in the temporal horn. The pars compacta of the substantia nigra was markedly thin and more rostrally, completely depigmented. The locus caeruleus in the pons was also severely depigmented. Histochemistry showed severe neuronal loss in the frontal and temporal cortices, and to a lesser extent in the parietal cortex with spongiosis and astrocytic gliosis (Fig. 4f). There was a severe atrophy of the hippocampus, amygdala and parahippocampal gyrus. The superior temporal gyrus was severely gliotic, with a thinning of the cortex to $1.5 \mathrm{~mm}$. The dorsomedial formation of the thalamus was affected with neuronal cell loss and gliotic changes and severe neuronal loss was observed in the substantia nigra. Neurofibrillary tangles, neuropil threads and dystrophic neurites is found in every cortical sample examined, as well as the thalamus, neostriatum, corpus mamillare, medial geniculate body, substantia nigra, and reticular formation of mesencephalon, pons and medulla oblongata (Fig. $4 \mathrm{~g}$ ). The lesion load was compatible with that of stage VI (Braak \& Braak) [57], and with a stage B3 of Montine et al. [56]. Classical and diffuse senile plaques were present in frontal, temporal, parietal and striatal cortices, as well as in the thalamus, putamen, medial geniculate body, 
corpus mamillare and in the molecular layer of the cerebellar cortex (Fig 4h). Cerebral amyloid angiopathy was mild [58] and the $\beta$-amyloid pathology was compatible with Phase 5 of Thal et al. [59], whereas the load of classical senile plaques was severe, compatible with CERAD stage 3 [60]. These findings are compatible with AD neuropathological changes A3B3C3 [56]. Both the TDP-43 and FUS stainings were negative. a-synuclein staining showed a moderate amount of Lewy bodies in the hippocampus and parahippocampal gyrus, and a severe amount in the amygdala. Sparse Lewy bodies were found in prefrontal cortex, the substantia nigra, the pons and the medulla oblongata (Fig. 4l-m). Based on these observations, patient $\mathrm{P} 3$ received a neuropathological diagnosis of $\mathrm{LBD}$, amygdala predominant type, and of AD neuropathological changes A3B3C3 $[7,56]$.

\section{Discussion}

VPS13C is a risk gene for PD patients identified in a meta-analysis of genome-wide association studies (GWAS) with an estimated odds ratio of $1.1[16,61,62]$. However, Lesage and colleagues identified homozygous and compound heterozygous PTC mutations in VPS13C in patients with a distinct form of early-onset parkinsonism, characterized by rapid and severe disease progression and early cognitive decline (Fig. 1b) [18]. The presence of Lewy bodies in the brainstem, limbic system and many cortical areas in one of the PD patients was reminiscent of diffuse LBD [18]. Later, a diagnostic WES data analysis of 80 persons with PD symptoms at early age and a negative family history, identified compound heterozygous variants affecting canonical splice sites in VPS13C in one early-onset PD patient (Fig. 1b) [19]. This patient presented overall with milder motor symptoms and disease progression, but presented with a rapid deterioration of cognitive functioning [19]. Shortly after, a large homozygous deletion comprising 50 exons of VPS13C was identified by WGS data of an sporadic patient with sensorimotor polyneuropathy and early-onset parkinsonism (Fig. 1b) [20]. This patient presented with normal cognitive functioning and a milder disease severity. Taken together, autosomal recessive loss of function mutations in VPS13C are rare and associated with early-onset PD with a high probability of cognitive deterioration and suggestive diffuse LBD pathology.

In contrast to the loss-of-function (LOF) mutations due to PTC mutations or deletions, we report for the first time that some combination of rare missense variants (MAF $\leq 1 \%)$ in VPS13C can also contribute to DLB and PD by loss-offunction mechanism and as such mimicking recessive inheritance. Our findings in family A triggered our interest in the role of rare missense variants in VPS13C and risk for LBD. In this family of healthy parents with two siblings affected with DLB at age early 41-42, we aimed at identifying the underlying genetic etiology using WGS. Taking that the pedigree suggested a potential recessive inheritance pattern, we searched for multiple rare variants in the same gene. The results indicated that rare compound heterozygous missense variants could explain the genetics in family $A$, with the patients combining 2 rare variants, p.Trp395Cys/p.Ala444Pro, inherited from their parents.

To expand our findings in family A, we screened the complete VPS13C coding region for multiple rare variants in the Belgian LBD cohort comprising DLB and PD patients and in controls. We observed a significant association $(p=0.0233)$ of rare (MAF $\leq 1 \%)$ variants and LBD patients. The full burden test including all genetic variants in VPS13C was not significant $(p=0.175)$. VPS13C is likely to contain deleterious, protective and null variants. Recently, a VPS13C haplotype including the common (MAF > 1\%) variants p.R153H-p.I398I-p.I1132V-p.Q2376Q was associated with a reduced risk for PD $(p=0.0052$, odds ratio $=0.48,95 \%$ confidence interval $=0.28-0.82)$ [63]. The same study, which included 1.567 lateonset PD patients, did not observe a statistically significant burden of rare (MAF $\leq 1 \%)$ VPS13C variants in PD, neither biallelic, non-synonymous coding and splice site variants in VPS13C in patients [63]. However, this study only included compound heterozygous variants with a MAF $<0.1 \%$ in late-onset patients, while we included variants with a MAF $<1 \%$ in both early- and late-onset patients. An independent study, investigating 4476 sporadic PD patients (mean AAO 60 years) and 5140 healthy control individuals reported a significant association $(p=0.002296)$ of rare (MAF $\leq 1 \%)$ VPS13C variants in PD [64]. 
In our Belgian LBD cohort, we identified 5 patients (4 DLB and 1 PD) with homozygous or trans compound heterozygous rare variants in VPS13C, including the patient of family A. We also observed 4 patients (1 DLB and 3 PD) with compound heterozygous variants in VPS13C with unknown phase $(9 / 844 ; 1.07 \%)$. In the control cohort, we observed two carriers $(2 / 664 ; 0.30 \%)$ of compound heterozygous VPS13C variants of unknown phase (Table S6). We were unable to phase the VPS13C alleles in the 4 patients and 2 controls (Table 1, Table S6). DNA of relatives for segregation or biomaterials for RNA isolation were not available for the carriers P8, P9 and C1. Long-read ONT cDNA sequencing, could not call the missense variants in P8 and P9. In C2, the VPS13C c. $448+7 A>G$ splice site variant is present downstream of exon 6 of VPS13C isoform 2 (NM_020821). We were not able to amplify VPS13C amplicons, containing exon 6 or 7 specific for isoform 2, from cDNA derived of lymphoblast cells, preventing phasing of c. $448+7 \mathrm{~A}>\mathrm{G} / \mathrm{p}$.Ala1687Val and evaluation of the effect of $c .448+7 A>G$ on splicing. We confirmed cis configuration of the compound heterozygous alleles in 3 patient and in 5 control carriers. This cis configuration is not fitting recessive inheritance and we excluded the carriers from further analyses in this study.

DLB patients P1 with compound heterozygous variants p.Trp395Cys/p.Ala444Pro, P2 with homozygous p.Ala444Pro and P3 with p.Thr1218Ala/p.lle2789Thr presented with a severe disease progression (Table S1). Neuropathological examination of patient carriers P1 and P3 indicated Lewy bodies in multiple brain regions (Fig. 4i-n). Since there was also extensive $A D$ pathology in patient $P 3$, there is a small likelihood that the pathological findings are associated with a DLB clinical syndrome. Nevertheless, both our clinical and neuropathological data of VPS13C mutation carriers supports the phenotype of most VPS13C patient carriers reported to date, including an early-onset age, severe disease progression and the co-occurrence of parkinsonism and dementia (Table 1, Table S1, ) [18-20].

Our genetic data suggest an enrichment of homozygous or compound VPS13C mutation carriers in patients compared to control though not significant $(p=0.1258)$, possibly because of the small numbers in the Belgian cohorts. We estimated the number of subjects needed for an adequate study power, taking into account the low frequency of compound carriers, showed that a minimum of 4200 subjects ( 2100 per patient/control cohort) would be required to reach $80 \%$ power. Replication of PTC mutations and missense variants in VPS13C in larger cohorts is necessary as well as the confirmation of trans configuration of the compound heterozygous variants to identify the carriers that can contribute to recessive inheritance.

The variability in onset age and the presence of potential compound heterozygotes in the control group can be explained by variable penetrance of the VPS13C mutant alleles might be explained by variability of loss of protein expression and functioning of the different mutant VPS13C alleles. In the patients P1 (family A) and P2, with bi-allelic p.Trp395Cys and/or p.Ala444Pro (Table 1, Fig. 2d), was the reduction of VPS13C protein expression in lymphoblast cells the most severe. Both patients developed the disease at very early age (40-42 years) and had a marked severe disease progression (Table S1). In brain tissue of the two patients, P1 and P3 (family B), with confirmed trans compound heterozygous VPS13C variants, VPS13C protein expression was remarkably reduced compared to control individuals, with the strongest reduction observed for patient P1 (Fig. 2f). The VPS13C mutant alleles of DLB patient P3 were present in one younger sibling II.4 (Family B; Fig. 1b). This sibling's age 62 is close to the onset age of the index patient P3 with a current age of 61 indicating that this sibling is still at risk for disease.

The human VPS13 family consists of four proteins, VPS13A/Chorein, VPS13B, VPS13C and VPS13D, with all family members having a strong homology to yeast Vps13. Yeast studies have suggested that Vps13 may have a role in lipid exchange between organelles and showed that yeast mutants lacking Vps13 cause defects in mitochondrial membrane integrity $[65,66]$. In mammalian cell models, VPS13C was suggested to partially localize to the mitochondrial outer membrane and silencing VPS13C results in a lower mitochondrial membrane potential, mitochondrial fragmentation and increased respiration rates [18]. However, recent research showed that human VPS13C functions as a tether between the ER and late endosomes/lysosomes, and between the ER and lipid droplets, enabling transport of glycerolipids between

Page $15 / 27$ 
membranes [54]. We confirmed the localization of wild type VPS13C at late endosomes and lysosomes (Fig. 3, Fig. S6, Fig. S7, Fig. S8). Overexpressing wild type or mutant VPS13C, containing p.Trp395Cys or p.Ala444Pro, in HeLa and SHSY5Y cells demonstrated that the late endosomal/lysosomal localization of VPS13C is completely lost in mutants (Fig. 3, Fig. S6, Fig. S7, Fig. S8). Surprisingly, both variants are located in the VPS13a domain and not in the putative WD40 modules responsible for endosomal/lysosomal localization (Fig. 1b). Because these variants are nearby the FFATmotif required for interaction with the ER, they may directly affect the localization to ER-contact sites as well. Overall, the different domains might be required for the protein's structural stability, needed for its localization to ER-late endosome/lysosome and ER-lipid droplet contact sites. Variants within the VPS13a domain may overall negatively affect the stability of the protein thereby affecting its localization. Besides p.Trp395Cys or p.Ala444Pro, we identified 12 alleles in compound heterozygous patient carriers mimicking recessive inheritance, including 11 missense and one splice site variants (Fig. 1b; Table 1), awaiting further functional investigation to estimate their pathogenicity.

Loss-of-function mutations in the other human VPS13 genes are associated with different recessive neurodegenerative or neurodevelopmental disorders. Mutations in VPS13A or Chorein cause a progressive neurodegenerative disorder, chorea-acanthocytosis, primarily characterized by chorea and red blood cell acanthocytosis, but may also include parkinsonism, dystonia frontal and cognitive impairment [67]. VPS13B is associated with Cohen syndrome, a clinically variable syndromic neurodevelopmental disorder with symptoms of microcephaly, intellectual disability and motor delay amongst others other features [68] whereas mutations in VPS13D cause a form of cerebellar ataxia with spasticity, which also appears to lead to mitochondrial dysfunction [69].

\section{Conclusions}

Overall, our results indicate that homozygous and compound heterozygous variants in VPS13C are associated with increased risk for LBD. We identified missense variants, i.e. p.Trp395Cys and p.Ala444Pro in VPS13C, with loss of functional protein confirming their pathogenicity. Understanding the contribution of the different mutated VPS13C alleles to the genetic etiology of LBD, needs additional research.

\section{Abbreviations}

AAl: Age at inclusion;

AAO: Age at onset age;

AD: Alzheimer's disease;

CADD: Combined Annotation Dependent Depletion;

CCN6, Cellular Communication Network Factor 6;

CLLU1, Chronic Lymphocytic Leukemia Up-regulated 1;

DLB: Dementia with Lewy bodies;

ER: Endoplasmic reticulum;

FTD: Frontotemporal dementia;

GWAS: Genome wide association studies;

IGV: Integrative Genomics Viewer; 
LBD: Lewy body disease;

MAF: Minor allele frequency;

MoCA: Montreal Cognitive Assessment;

ONT, Oxford Nanopore Technologies;

PD: Parkinson's disease;

PMD, Post mortem delay;

PTC: Premature termination codon;

REM: Rapid eye movement; RNF6, Ring Finger Protein 6;

SKAT-O: Optimized sequence kernel association test;

VPS13C: Vacuolar Protein Sorting 13 homolog C;

WGS: Whole genome sequencing;

WT: Wild type.

\section{Declarations}

\section{Ethics approval and consent to participate}

The local ethical committees of the collaborating hospitals approved the protocols for the clinical studies and the informed consent form. The ethic committee of the University Hospital of Antwerp and University of Antwerp approved the genetic studies and informed consent form.

\section{Consent for publication}

Not applicable.

\section{Availability of data and materials}

All data relevant to this study are included in the research paper or added to the supplementary information. The corresponding author will share additional information upon reasonable request.

\section{Competing interests}

None of the authors reported personal competing financial interests.

\section{Funding}


In part funded by the Belgian Science Policy Office Interuniversity Attraction Poles program, the Flemish Government initiated Flanders Impulse Program on Networks for Dementia Research and the Methusalem Excellence Program, the Research Foundation Flanders (FWO), the University of Antwerp Research Fund, the KU Leuven research council and VIB. W.A. received support of the Fund Bonmariage de Bouyalski by the King Baudouin Foundation and the SAO-FRA Research Foundation for Alzheimer's disease; Belgium.

\section{Authors' contributions}

S.S. conceived the design of the studies, performed targeted resequencing of the LBD cohort and the experiments to determine cis/trans position of the variants, participated in the functional work, analyzed the data, performed statistical analysis, drafted the manuscript and created figures. S.P. analyzed the whole genome sequencing data, composed the MASTR assay for VPS13C resequencing, assisted in targeted resequencing of the control cohort and the functional work and created figures. B.H. assisted in Western blotting and created figures. B.A. assisted in the design and execution of the cell live imaging and immunohistochemistry and created the figures. RDSD generated and provided polyclonal VPS13C antibody. A.S., Y.V., and J-J.M. provided neuropathological information of autopsy patients. D.C., E.H., P.P., R.V., S.E., P.P.D.D. and P.C. provided patient blood samples and clinical information. W.A. provided VPS13C antibodies, advised for the functional experiments and support for the paper. C.V.B. conceived and oversighted the project and was involved in the design of the project and studies, data interpretation, writing of the paper and critical revision of the manuscript. All authors reviewed the manuscript.

\section{Acknowledgements}

The authors acknowledge the contribution of the following neurologists, members of the BELNEU consortium: sampling of PD and DLB patients, performing clinical and pathological phenotyping and follow-up of patients and families: Chris van der Linden (General Hospital St. Lucas Ghent, Ghent); Emke Maréchal (Antwerp Hospital Network, Antwerp); Rik Vandenberghe (University Hospitals Leuven, Leuven) and Bruno Bergmans (General Hospital Sint-Jan, Brugge).

The authors thank the personnel of the Neuromics Support Facility of the VIB Center for Molecular Neurology, the DNA Screening Facility and Biobank of the Neurodegenerative Brain Diseases group, the NeuroBiobank of the Institute BornBunge and the neurological centers of the BELNEU Consortium partners. The authors recognize the assistances of the technicians, Nathalie Geerts and Ivy Cuijt, for the genetic screenings and functional experiments in this project.

\section{Table}

Table 1: LBD patients carrying rare homozygous or heterozygous compound variants in VPS13C. 


\begin{tabular}{|c|c|c|c|c|c|c|c|c|c|c|c|}
\hline Patient & $\mathrm{Dx}$ & AAO & $\Delta \mathrm{CDS}^{\mathrm{a}}$ & $\Delta \mathrm{AA}^{\mathrm{b}}$ & $\begin{array}{l}\text { MAF } \\
\text { gnomAD } \\
(\%) \\
{[\text { Hom] }}\end{array}$ & $\begin{array}{l}\text { CADD_Phred } \\
\text { score }^{\text {c }}\end{array}$ & $\begin{array}{l}\text { MAF } \\
\text { patient } \\
\text { cohort } \\
(\%) \\
\mathrm{n}=844 \\
\end{array}$ & $\begin{array}{l}\text { MAF } \\
\text { control } \\
\text { cohort } \\
(\%) \\
\mathrm{n}=664 \\
\end{array}$ & $\begin{array}{l}F \text { in } \\
\text { patients } \\
(\%)\end{array}$ & $\begin{array}{l}\mathrm{F} \text { in } \\
\text { controls } \\
(\%)\end{array}$ & $\begin{array}{l}\text { F } \\
\text { expected }^{d} \\
(\%)\end{array}$ \\
\hline \multirow[t]{2}{*}{$\mathrm{P} 1 *, \$$} & DLB & 42 & c. $1185 \mathrm{G}>\mathrm{C}$ & p.Trp395Cys $\$$ & - & 33 & 0.0592 & 0 & 0.118 & 0 & 0.0000770 \\
\hline & & & c. $1330 \mathrm{G}>\mathrm{C}$ & p.Ala444Pro ${ }^{\S}$ & $\begin{array}{l}0.00864 \\
{[0]}\end{array}$ & 28.4 & 0.415 & 0 & & & \\
\hline \multirow[t]{2}{*}{$\mathrm{P} 2 *$} & DLB & 40 & c. $1330 \mathrm{G}>\mathrm{C}$ & p.Ala444Pro ${ }^{\S}$ & $\begin{array}{l}0.00864 \\
{[0]}\end{array}$ & 28.4 & 0.415 & 0 & 0.118 & 0 & 0.0005 \\
\hline & & & c. $1330 \mathrm{G}>\mathrm{C}$ & p.Ala444Pro ${ }^{\S}$ & $\begin{array}{l}0.00864 \\
{[0]}\end{array}$ & 28.4 & 0.415 & 0 & & & \\
\hline \multirow[t]{2}{*}{$\mathrm{P} 3 *, \$$} & DLB & 58 & c. $3652 A>G$ & p.Thr1218Ala & $\begin{array}{l}0.000781 \\
{[0]}\end{array}$ & 26.4 & 0.0592 & 0 & 0.118 & 0 & 0.00020 \\
\hline & & & c.8366T $>C$ & p.Ile2789Thr ${ }^{\S}$ & $1.00[7]$ & 2.797 & 0.533 & 0.679 & & & \\
\hline \multirow[t]{2}{*}{ P4 } & DLB & 84 & c. $8133 \mathrm{G}>\mathrm{A}$ & p.Met2711Ile & $\begin{array}{l}0.00177 \\
{[0]}\end{array}$ & 22.5 & 0.0592 & 0.0754 & 0.118 & 0 & 0.000396 \\
\hline & & & c.8366T $>C$ & p.Ile2789Thr ${ }^{\S}$ & $1.00[7]$ & 2.797 & 0.533 & 0.679 & & & \\
\hline \multirow[t]{2}{*}{ P5* } & $\mathrm{PD}$ & 60 & c. $5060 \mathrm{C}>\mathrm{T}$ & p.Ala1687Val ${ }^{\S}$ & $0.314[0]$ & 22.5 & 0.0592 & 0.151 & 0.118 & 0 & 0.000264 \\
\hline & & & c. $8711 \mathrm{C}>\mathrm{T}$ & p.Ser2904Leu ${ }^{\S}$ & $0.343[0]$ & 26.7 & 0.178 & 0.378 & & & \\
\hline \multirow[t]{2}{*}{ P6* } & DLB & 42 & c. $2797 \mathrm{~A}>\mathrm{G}$ & p.Thr933Ala & $0.577[2]$ & 12.67 & 0.711 & 0.452 & 0.118 & 0 & 0.000991 \\
\hline & & & c. $7276 \mathrm{G}>\mathrm{A}$ & p.Val2426Ile & $0.244[0]$ & 12.31 & 0.118 & 0.226 & & & \\
\hline \multirow[t]{2}{*}{$\mathrm{P} 7 *$} & PD & 65 & c. $2296 \mathrm{~A}>\mathrm{G}$ & p.Thr766Ala & - & 12.18 & 0.0592 & 0.0754 & 0.118 & 0 & 0.0000220 \\
\hline & & & c. $5537 \mathrm{~T}>\mathrm{C}$ & p.Leu1846Ser & - & 26.8 & 0.0592 & 0 & & & \\
\hline \multirow[t]{2}{*}{ P8 } & $\mathrm{PD}$ & 73 & c. $4056+3 \mathrm{~A}>\mathrm{C}$ & - & $\begin{array}{l}0.00705 \\
{[0]}\end{array}$ & 14.06 & 0.0592 & 0 & 0.118 & 0 & 0.0000110 \\
\hline & & & c. $7628 \mathrm{C}>\mathrm{G}$ & p.Ser2543Cys & - & 28.9 & 0.0592 & 0 & & & \\
\hline \multirow[t]{2}{*}{ P9 } & $\mathrm{PD}$ & 73 & c. $8366 \mathrm{~T}>\mathrm{C}$ & p.Ile2789Thr & $1.00[7]$ & 2.797 & 0.533 & 0.679 & 0.118 & 0 & 0.00337 \\
\hline & & & c. $11176 \mathrm{~A}>\mathrm{G}$ & p.Ile3726Val & $0.594[5]$ & 23.1 & 0.237 & 0.980 & & & \\
\hline
\end{tabular}

Note: Clinical symptoms of DLB and PD patients summarized in Table S1. *Lymphoblast cells and ${ }^{\text {\$brain }}$

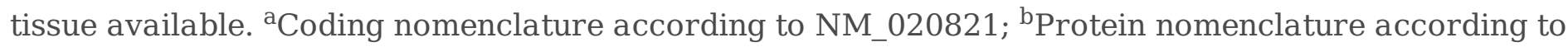
NP_0658721; 'CADD_Phred, score [42]. d Expected frequency is calculated according to the HardyWeinberg principle, using the MAF of the single alleles in patients plus controls $(\mathrm{n}=1508)$; ${ }^{\S}$ Variants in trans configuration; Abbreviations: Dx diagnosis; $\Delta$ CDS coding sequence substitution; $\Delta \mathrm{AA}$ amino acid substitution, MAF, minor allele frequency, F frequency of homozygotes and compound heterozygotes; AAO, age at onset; gnomAD, Genome Aggregation Database [70]; Hom, Number of homozygotes in gnomAD.

\section{References}

1. Walker L, Stefanis L, Attems J. Clinical and neuropathological differences between Parkinson's disease, Parkinson's disease dementia and dementia with Lewy bodies - current issues and future directions. J Neurochem. 2019;150(5):467-74.

2. Galasko D. Lewy Body Disorders. Neurol Clin. 2017;35(2):325-38.

3. Mok W, Chow TW, Zheng L, Mack WJ, Miller C. Clinicopathological concordance of dementia diagnoses by community versus tertiary care clinicians. Am J Alzheimers Dis Other Demen. 2004;19(3):161-5.

4. Kang JH, Korecka M, Toledo JB, Trojanowski JQ, Shaw LM. Clinical utility and analytical challenges in measurement of cerebrospinal fluid amyloid-beta(1-42) and tau proteins as Alzheimer disease biomarkers. Clin Chem.

2013;59(6):903-16. 
5. Meeus B, Verstraeten A, Crosiers D, Engelborghs S, Van den Broeck M, Mattheijssens $M$, et al. DLB and PDD: a role for mutations in dementia and Parkinson disease genes? Neurobiol Aging. 2012;33(3):629 e5- e18.

6. Vann Jones SA, O'Brien JT. The prevalence and incidence of dementia with Lewy bodies: a systematic review of population and clinical studies. Psychol Med. 2014;44(4):673-83.

7. McKeith IG, Dickson DW, Lowe J, Emre M, O'Brien JT, Feldman H, et al. Diagnosis and management of dementia with Lewy bodies: third report of the DLB Consortium. Neurology. 2005;65(12):1863-72.

8. Toledo JB, Gopal P, Raible K, Irwin DJ, Brettschneider J, Sedor S, et al. Pathological alpha-synuclein distribution in subjects with coincident Alzheimer's and Lewy body pathology. Acta Neuropathol. 2016;131(3):393-409.

9. Pringsheim T, Jette N, Frolkis A, Steeves TD. The prevalence of Parkinson's disease: a systematic review and metaanalysis. Mov Disord. 2014;29(13):1583-90.

10. Aarsland D, Zaccai J, Brayne C. A systematic review of prevalence studies of dementia in Parkinson's disease. Mov Disord. 2005;20(10):1255-63.

11. Braak H, Del Tredici K, Rub U, de Vos RA, Jansen Steur EN, Braak E. Staging of brain pathology related to sporadic Parkinson's disease. Neurobiol Aging. 2003;24(2):197-211.

12. Meeus B, Nuytemans $K$, Crosiers D, Engelborghs S, Peeters $K$, Mattheijssens $M$, et al. Comprehensive genetic and mutation analysis of familial dementia with Lewy bodies linked to 2q35-q36. J Alzheimers Dis. 2010;20(1):197-205.

13. Bogaerts V, Engelborghs S, Kumar-Singh S, Goossens D, Pickut B, van der Zee J, et al. A novel locus for dementia with Lewy bodies: a clinically and genetically heterogeneous disorder. Brain. 2007;130(Pt 9):2277-91.

14. Bonner LT, Tsuang DW, Cherrier MM, Eugenio CJ, Du Jennifer Q, Steinbart EJ, et al. Familial dementia with Lewy bodies with an atypical clinical presentation. J Geriatr Psychiatry Neurol. 2003;16(1):59-64.

15. Blauwendraat C, Nalls MA, Singleton AB. The genetic architecture of Parkinson's disease. Lancet Neurol. 2019.

16. Nalls MA, Blauwendraat C, Vallerga CL, Heilbron K, Bandres-Ciga S, Chang D, et al. Identification of novel risk loci, causal insights, and heritable risk for Parkinson's disease: a meta-analysis of genome-wide association studies. Lancet Neurol. 2019;18(12):1091-102.

17. Martin S, Smolders S, Van den Haute C, Heeman B, van Veen S, Crosiers D, et al. Mutated ATP10B increases Parkinson's disease risk by compromising lysosomal glucosylceramide export. Acta Neuropathol. 2020.

18. Lesage S, Drouet V, Majounie E, Deramecourt V, Jacoupy M, Nicolas A, et al. Loss of VPS13C Function in AutosomalRecessive Parkinsonism Causes Mitochondrial Dysfunction and Increases PINK1/Parkin-Dependent Mitophagy. Am J Hum Genet. 2016;98(3):500-13.

19. Schormair B, Kemlink D, Mollenhauer B, Fiala O, Machetanz G, Roth J, et al. Diagnostic exome sequencing in earlyonset Parkinson's disease confirms VPS13C as a rare cause of autosomal-recessive Parkinson's disease. Clin Genet. 2018;93(3):603-12.

20. Darvish H, Bravo P, Tafakhori A, Azcona LJ, Ranji-Burachaloo S, Johari AH, et al. Identification of a large homozygous VPS13C deletion in a patient with early-onset Parkinsonism. Mov Disord. 2018;33(12):1968-70.

21. Creese B, Bell E, Johar I, Francis P, Ballard C, Aarsland D. Glucocerebrosidase mutations and neuropsychiatric phenotypes in Parkinson's disease and Lewy body dementias: Review and meta-analyses. Am J Med Genet B Neuropsychiatr Genet. 2018;177(2):232-41.

22. Ikeuchi T, Kakita A, Shiga A, Kasuga K, Kaneko H, Tan CF, et al. Patients homozygous and heterozygous for SNCA duplication in a family with parkinsonism and dementia. Arch Neurol. 2008;65(4):514-9.

23. Konno T, Ross OA, Puschmann A, Dickson DW, Wszolek ZK. Autosomal dominant Parkinson's disease caused by SNCA duplications. Parkinsonism Relat Disord. 2016;22 Suppl 1:S1-6.

24. Guerreiro R, Ross OA, Kun-Rodrigues C, Hernandez DG, Orme T, Eicher JD, et al. Investigating the genetic architecture of dementia with Lewy bodies: a two-stage genome-wide association study. Lancet Neurol. 2018;17(1):64-74.

Page 20/27 
25. Bras J, Guerreiro R, Darwent L, Parkkinen L, Ansorge O, Escott-Price V, et al. Genetic analysis implicates APOE, SNCA and suggests lysosomal dysfunction in the etiology of dementia with Lewy bodies. Hum Mol Genet. 2014;23(23):6139-46.

26. Gijselinck I, Van Langenhove T, van der Zee J, Sleegers K, Philtjens S, Kleinberger G, et al. A C9orf72 promoter repeat expansion in a Flanders-Belgian cohort with disorders of the frontotemporal lobar degeneration-amyotrophic lateral sclerosis spectrum: a gene identification study. Lancet Neurol. 2012;11(1):54-65.

27. Van Langenhove T, van der Zee J, Gijselinck I, Engelborghs S, Vandenberghe R, Vandenbulcke M, et al. Distinct clinical characteristics of C9orf72 expansion carriers compared with GRN, MAPT, and nonmutation carriers in a Flanders-Belgian FTLD cohort. JAMA Neurol. 2013;70(3):365-73.

28. McKeith IG, Boeve BF, Dickson DW, Halliday G, Taylor JP, Weintraub D, et al. Diagnosis and management of dementia with Lewy bodies: Fourth consensus report of the DLB Consortium. Neurology. 2017;89(1):88-100.

29. Gelb DJ, Oliver E, Gilman S. Diagnostic criteria for Parkinson disease. Arch Neurol. 1999;56(1):33-9.

30. Homig-Holzel C, Savola S. Multiplex ligation-dependent probe amplification (MLPA) in tumor diagnostics and prognostics. Diagn Mol Pathol. 2012;21(4):189-206.

31. Nuytemans K, Meeus B, Crosiers D, Brouwers N, Goossens D, Engelborghs S, et al. Relative contribution of simple mutations vs. copy number variations in five Parkinson disease genes in the Belgian population. Hum Mutat. 2009;30(7):1054-61.

32. Nasreddine ZS, Phillips NA, Bedirian V, Charbonneau S, Whitehead V, Collin I, et al. The Montreal Cognitive Assessment, MoCA: a brief screening tool for mild cognitive impairment. J Am Geriatr Soc. 2005;53(4):695-9.

33. Drmanac R, Sparks AB, Callow MJ, Halpern AL, Burns NL, Kermani BG, et al. Human genome sequencing using unchained base reads on self-assembling DNA nanoarrays. Science. 2010;327(5961):78-81.

34. Reumers J, De Rijk P, Zhao H, Liekens A, Smeets D, Cleary J, et al. Optimized filtering reduces the error rate in detecting genomic variants by short-read sequencing. Nat Biotechnol. 2012;30(1):61-8.

35. Smit A, Hubley R, Green P. RepeatMasker Open-3.0. 1996-2010.

36. Auton A, Brooks LD, Durbin RM, Garrison EP, Kang HM, Korbel JO, et al. A global reference for human genetic variation. Nature. 2015;526(7571):68-74.

37. Lange V, Bohme I, Hofmann J, Lang K, Sauter J, Schone B, et al. Cost-efficient high-throughput HLA typing by MiSeq amplicon sequencing. BMC Genomics. 2014;15:63.

38. Li H, Durbin R. Fast and accurate short read alignment with Burrows-Wheeler transform. Bioinformatics. 2009;25(14):1754-60.

39. Li H, Durbin R. Fast and accurate long-read alignment with Burrows-Wheeler transform. Bioinformatics. 2010;26(5):589-95.

40. McKenna A, Hanna M, Banks E, Sivachenko A, Cibulskis K, Kernytsky A, et al. The Genome Analysis Toolkit: a MapReduce framework for analyzing next-generation DNA sequencing data. Genome Res. 2010;20(9):1297-303.

41. DePristo MA, Banks E, Poplin R, Garimella KV, Maguire JR, Hartl C, et al. A framework for variation discovery and genotyping using next-generation DNA sequencing data. Nat Genet. 2011;43(5):491-8.

42. Kircher M, Witten DM, Jain P, O'Roak BJ, Cooper GM, Shendure J. A general framework for estimating the relative pathogenicity of human genetic variants. Nat Genet. 2014;46(3):310-5.

43. Robinson JT, Thorvaldsdottir H, Wenger AM, Zehir A, Mesirov JP. Variant Review with the Integrative Genomics Viewer. Cancer Res. 2017;77(21):e31-e4.

44. Rozen S, Skaletsky H. Primer3 on the WWW for general users and for biologist programmers. Methods Mol Biol. 2000;132:365-86. 
45. Weckx S, Del-Favero J, Rademakers R, Claes L, Cruts M, De Jonghe P, et al. novoSNP, a novel computational tool for sequence variation discovery. Genome Res. 2005;15(3):436-42.

46. Brouwers N, Nuytemans K, van der Zee J, Gijselinck I, Engelborghs S, Theuns J, et al. Alzheimer and Parkinson diagnoses in progranulin null mutation carriers in an extended founder family. Arch Neurol. 2007;64(10):1436-46.

47. Gijselinck I, van der Zee J, Engelborghs S, Goossens D, Peeters K, Mattheijssens M, et al. Progranulin locus deletion in frontotemporal dementia. Hum Mutat. 2008;29(1):53-8.

48. Schneider VA, Graves-Lindsay T, Howe K, Bouk N, Chen HC, Kitts PA, et al. Evaluation of GRCh38 and de novo haploid genome assemblies demonstrates the enduring quality of the reference assembly. Genome Res. 2017;27(5):849-64.

49. Li H. Minimap2: pairwise alignment for nucleotide sequences. Bioinformatics. 2018;34(18):3094-100.

50. Li H, Handsaker B, Wysoker A, Fennell T, Ruan J, Homer N, et al. The Sequence Alignment/Map format and SAMtools. Bioinformatics. 2009;25(16):2078-9.

51. Edge P, Bansal V. Longshot enables accurate variant calling in diploid genomes from single-molecule long read sequencing. Nat Commun. 2019;10(1):4660.

52. Schindelin J, Arganda-Carreras I, Frise E, Kaynig V, Longair M, Pietzsch T, et al. Fiji: an open-source platform for biological-image analysis. Nat Methods. 2012;9(7):676-82.

53. Velayos-Baeza A, Vettori A, Copley RR, Dobson-Stone C, Monaco AP. Analysis of the human VPS13 gene family. Genomics. 2004;84(3):536-49.

54. Kumar N, Leonzino M, Hancock-Cerutti W, Horenkamp FA, Li P, Lees JA, et al. VPS13A and VPS13C are lipid transport proteins differentially localized at ER contact sites. J Cell Biol. 2018;217(10):3625-39.

55. Deramecourt V, Slade JY, Oakley AE, Perry RH, Ince PG, Maurage CA, et al. Staging and natural history of cerebrovascular pathology in dementia. Neurology. 2012;78(14):1043-50.

56. Hyman BT, Phelps CH, Beach TG, Bigio EH, Cairns NJ, Carrillo MC, et al. National Institute on Aging-Alzheimer's Association guidelines for the neuropathologic assessment of Alzheimer's disease. Alzheimers Dement. 2012;8(1):113.

57. Braak H, Braak E. Neuropathological stageing of Alzheimer-related changes. Acta Neuropathol. 1991;82(4):239-59.

58. Love S, Chalmers K, Ince P, Esiri M, Attems J, Jellinger K, et al. Development, appraisal, validation and implementation of a consensus protocol for the assessment of cerebral amyloid angiopathy in post-mortem brain tissue. Am J Neurodegener Dis. 2014;3(1):19-32.

59. Thal DR, Rub U, Orantes M, Braak H. Phases of A beta-deposition in the human brain and its relevance for the development of AD. Neurology. 2002;58(12):1791-800.

60. Mirra SS, Heyman A, McKeel D, Sumi SM, Crain BJ, Brownlee LM, et al. The Consortium to Establish a Registry for Alzheimer's Disease (CERAD). Part II. Standardization of the neuropathologic assessment of Alzheimer's disease. Neurology. 1991;41(4):479-86.

61. Nalls MA, Pankratz N, Lill CM, Do CB, Hernandez DG, Saad M, et al. Large-scale meta-analysis of genome-wide association data identifies six new risk loci for Parkinson's disease. Nat Genet. 2014;46(9):989-93.

62. Chang D, Nalls MA, Hallgrimsdottir IB, Hunkapiller J, van der Brug M, Cai F, et al. A meta-analysis of genome-wide association studies identifies 17 new Parkinson's disease risk loci. Nat Genet. 2017;49(10):1511-6.

63. Rudakou U, Ruskey JA, Krohn L, Laurent SB, Spiegelman D, Greenbaum L, et al. Analysis of common and rare VPS13C variants in late-onset Parkinson disease. Neurology Genetics. 2020;6(1):385.

64. Hopfner F, Mueller SH, Szymczak S, Junge O, Tittmann L, May S, et al. Rare Variants in Specific Lysosomal Genes Are Associated with Parkinson's Disease. Mov Disord. 2020. 
65. Lang AB, John Peter AT, Walter P, Kornmann B. ER-mitochondrial junctions can be bypassed by dominant mutations in the endosomal protein Vps13. J Cell Biol. 2015;210(6):883-90.

66. Park JS, Thorsness MK, Policastro R, McGoldrick LL, Hollingsworth NM, Thorsness PE, et al. Yeast Vps13 promotes mitochondrial function and is localized at membrane contact sites. Mol Biol Cell. 2016;27(15):2435-49.

67. Danek A, Jung HH, Melone MA, Rampoldi L, Broccoli V, Walker RH. Neuroacanthocytosis: new developments in a neglected group of dementing disorders. J Neurol Sci. 2005;229-230:171-86.

68. Kolehmainen J, Black GC, Saarinen A, Chandler K, Clayton-Smith J, Traskelin AL, et al. Cohen syndrome is caused by mutations in a novel gene, $\mathrm{COH} 1$, encoding a transmembrane protein with a presumed role in vesicle-mediated sorting and intracellular protein transport. Am J Hum Genet. 2003;72(6):1359-69.

69. Seong E, Insolera R, Dulovic M, Kamsteeg EJ, Trinh J, Bruggemann N, et al. Mutations in VPS13D lead to a new recessive ataxia with spasticity and mitochondrial defects. Ann Neurol. 2018;83(6):1075-88.

70. Lek M, Karczewski KJ, Minikel EV, Samocha KE, Banks E, Fennell T, et al. Analysis of protein-coding genetic variation in 60,706 humans. Nature. 2016;536(7616):285-91.

\section{Figures}

a Family A

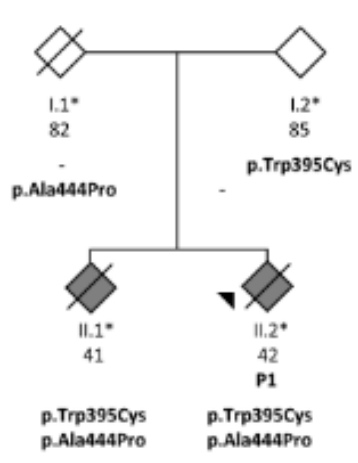

b

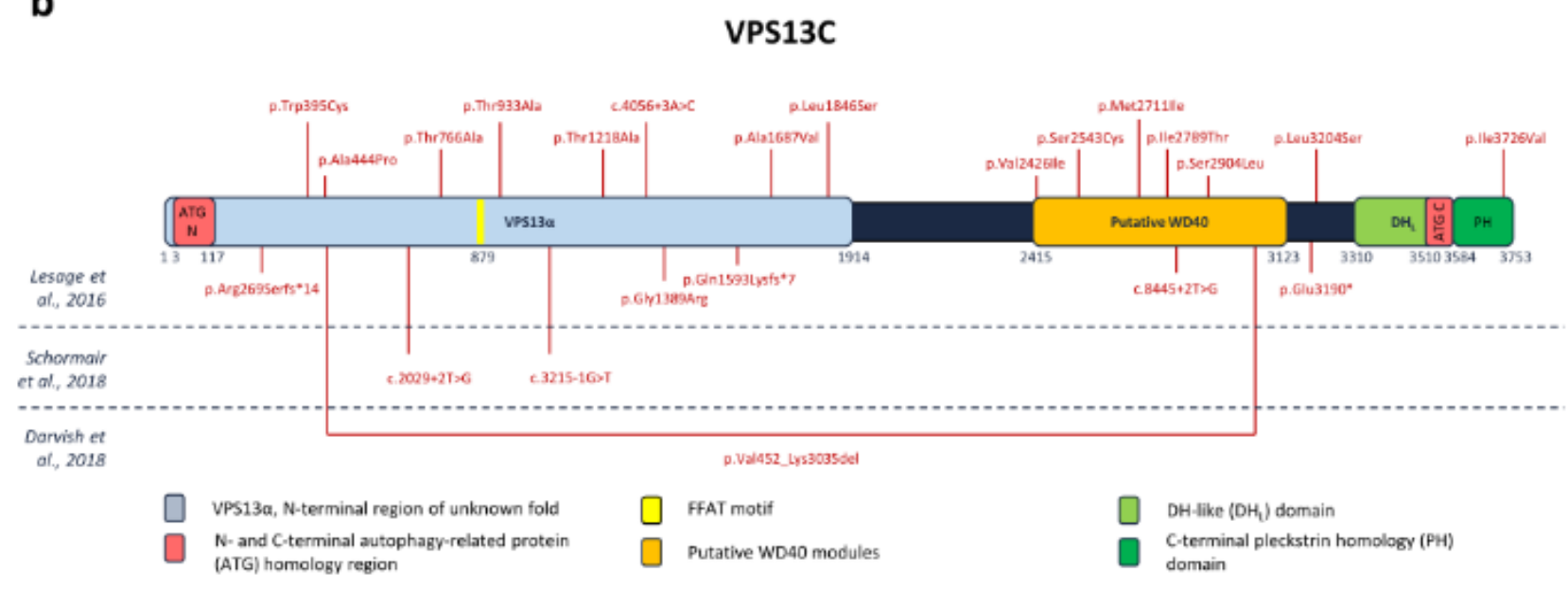

Family C

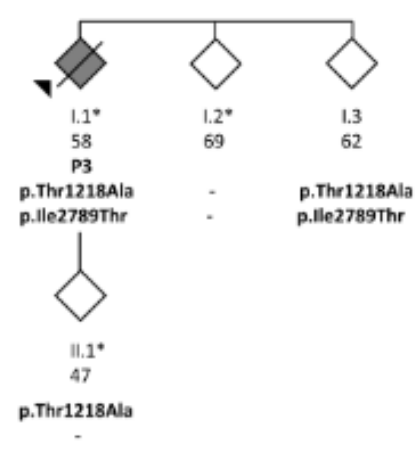

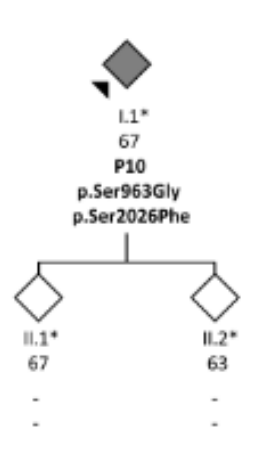

Figure 1 
VPS13C rare variants in 3 Belgian LBD families and in a LBD cohort. a. Pedigree of family A, B and C. Family A with two siblings affected with DLB and carriers of trans compound heterozygous rare variants, p.Trp395Cys/p.Ala444Pro. The index patient P2 (black arrow), received a pathological diagnosis of diffuse LBD of the neocortical type. Family $B$ with one affected patient (P3) with compound heterozygous variants, p.Thr1218Ala/p.Ile2789Thr. Patient P3 had a pathological diagnosis of LBD, of the predominant amygdala type. Additionally, patient P3 had full AD pathology in all three neuropathological changes, A3B3C3 $[7,56]$. Family $C$ with one affected patient (P10) with compound heterozygous VPS13C variants p.Ser963Gly and p.Ser2026Phe. Both children of patient P10 are negative for p.Ser963Gly and p.Ser2026Phe, suggesting cis configuration of the variants in the patient. To make the pedigrees anonymous, we used diamonds for the family members and the patients (black symbol) and we added to the pedigree only family members needed to show the cis/trans location of the VPS13C variants. Slashed symbols indicate deceased family members. $b$. Linear presentation of the VPS13C protein with domains based on [54], protein nomenclature according to NP_065872.1. Above, we positioned the homozygous or compound heterozygous variants with a MAF of $\leq 1 \%$, observed in the families and in the DLB and PD cohorts. The VPS13Ca domain is involved in transport of glycerophospholipids, the putative WD40 modules contain the binding site for late endosomes/lysosomes and the DH-like (DHL)-pleckstrin homology (PH) domains is the lipid droplet-binding region of VPS13C [54]. We showed published PTC mutations and deletions below the VPS13C protein, with at the left first author name and year of publication [18-20].

a

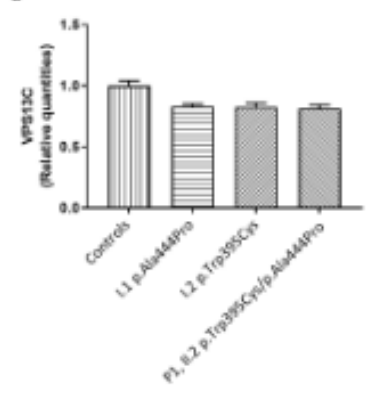

d

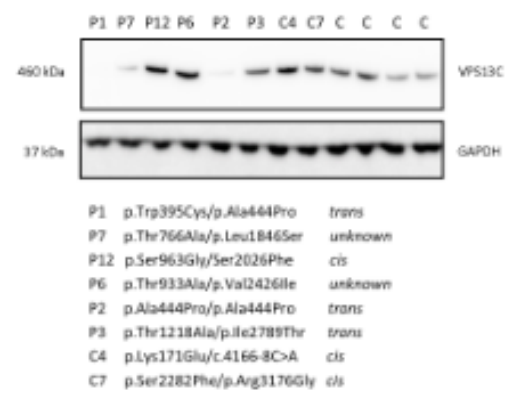

c

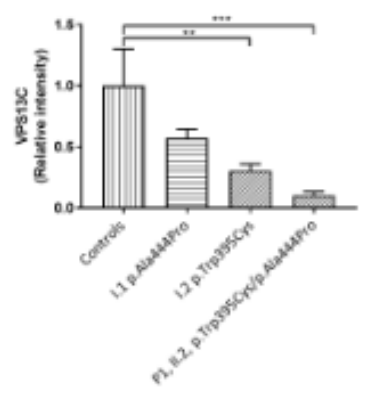

e

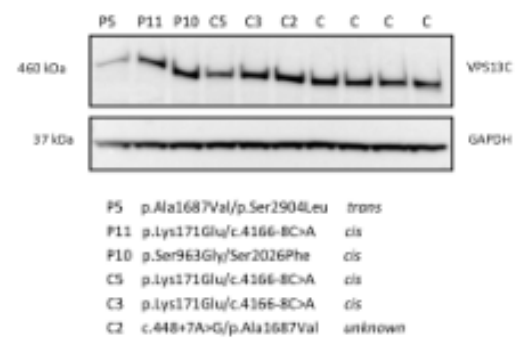

f
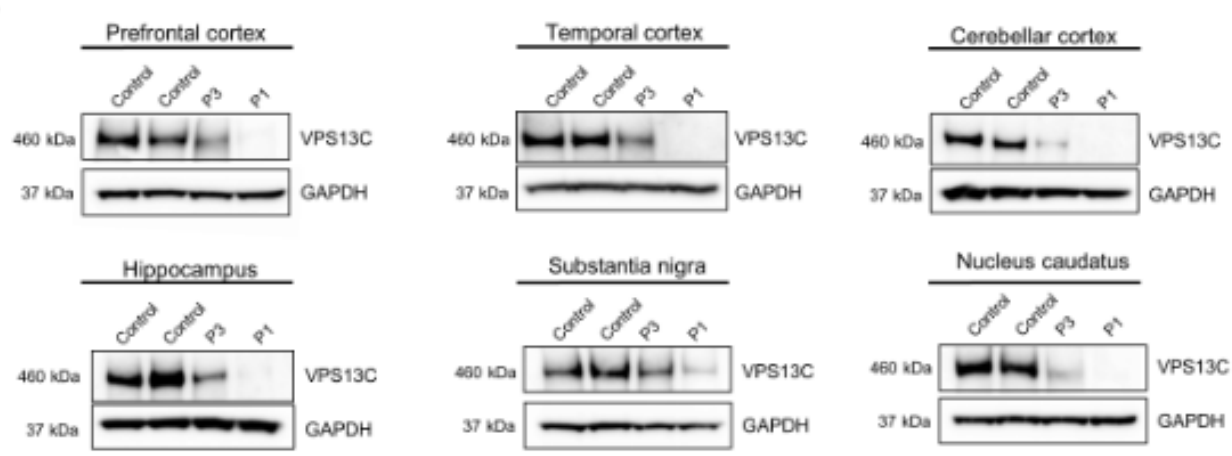

Figure 2

Reduced VPS13C protein expression in lymphoblast cells and brain lysates of mutation carriers. a. Relative VPS13C mRNA expression levels of family $A$ and unrelated control individuals $(n=3)$ in lymphoblast cells. b. Representative 
immunoblots of endogenous VPS13C expression in family A and unrelated control individuals $(n=3)$ in lymphoblast cells. c. Quantification of VPS13C protein expression, normalized with the expression of GAPDH in lymphoblast cells. Error bars represent standard deviation. d-e. Representative immunoblots of endogenous VPS13C protein expression in lymphoblast cells of patient and control carriers with $>1$ VPS13C variants, and unrelated control individuals $(n=4)$. $f$. Representative immunoblots of endogenous VPS13C protein expression in brains of patient carrier P1 and P3 and two unrelated control individuals. Protein levels were measured in the prefrontal cortex (d), the temporal cortex (e), the cerebellar cortex (f), the hippocampus (g), the substantia nigra (h), the caudate nucleus (i) and the putamen (j); $\star * 0.001<\mathrm{P}<0.01 ; * \star * 0.0001<\mathrm{P}<0.001 ; * \star * * \mathrm{P}<0.0001$

a

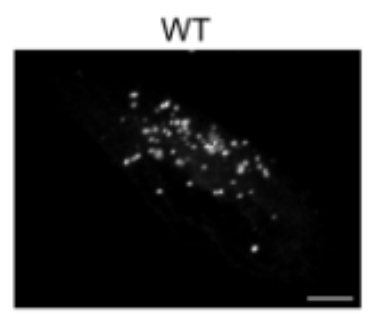

C
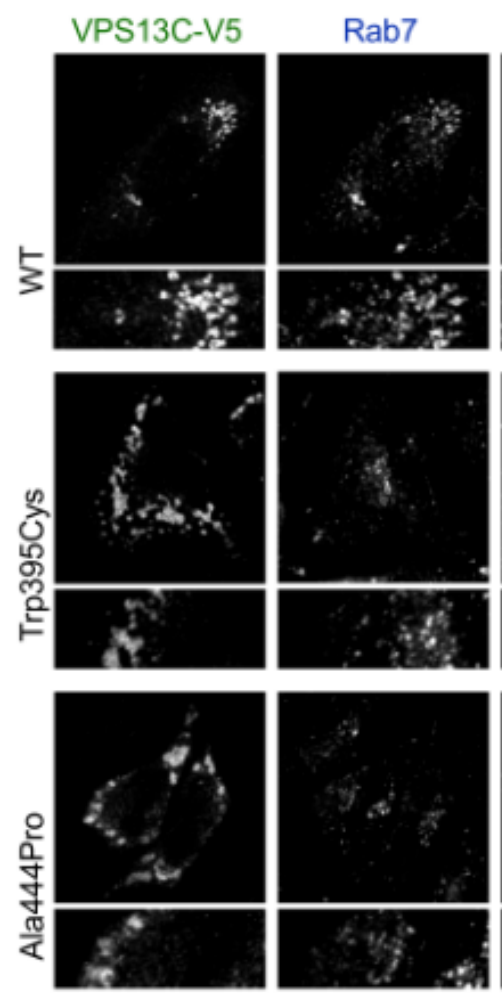

Mutant

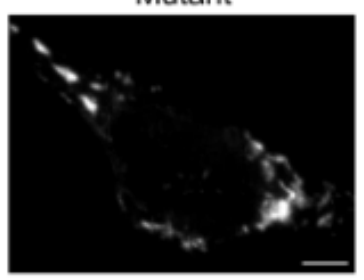

b

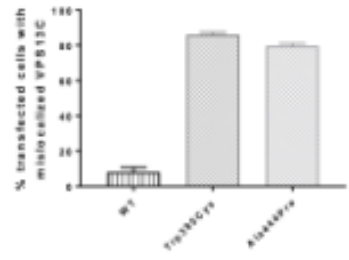

vpstse vianense mutation
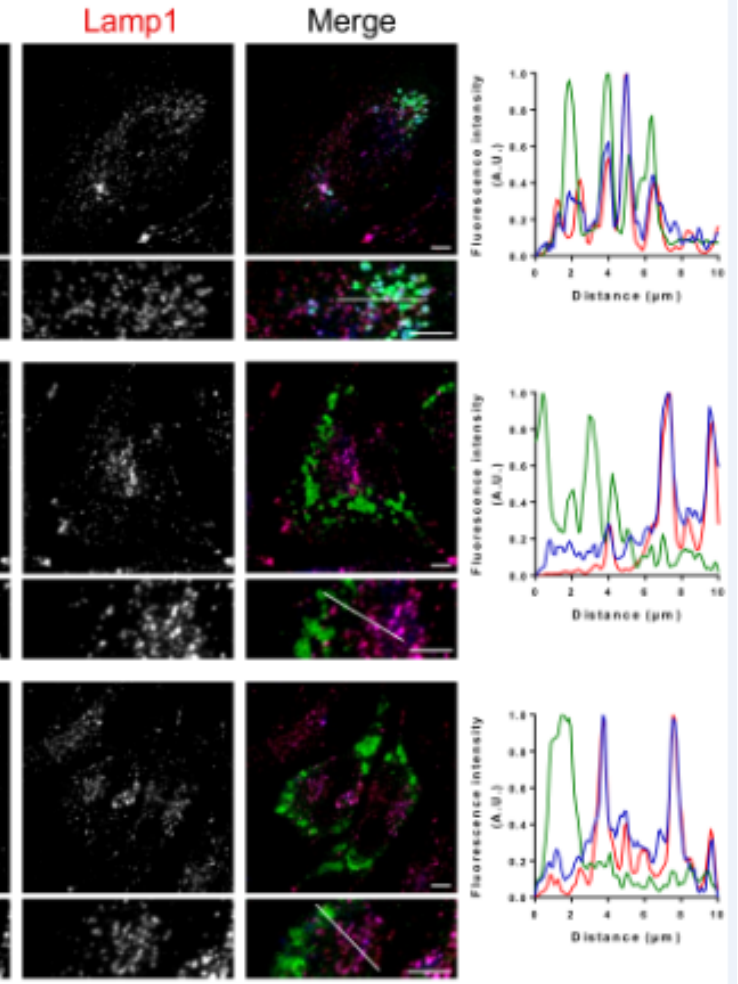

\section{Figure 3}

Localization of VPS13C at the late endosomes and lysosomes disturbed by p.Trp395Cys and p.Ala444Pro. Triple immunofluorescence of V5 (VPS13C constructs, green) with markers for late endosomes (Rab7) and lysosomes (Lamp1) in HeLa cells. a. Cells expressing VPS13C WT showed a clear vesicular staining pattern while those expressing the missense mutations p.Trp395Cys or p.Ala444Pro mislocalized in larger cytosolic structures. b. Quantification of cells that appeared either vesicular or mislocalized. c. Staining with anti-V5 antibody in WT-overexpressing HeLa cells showed co-staining with markers against late endosomes and lysosomes. HeLa cells overexpressing either p.Trp395Cys or p.Ala444Pro showed no co-localization with endosomes and lysosomes. White lines indicate the position of the intensity profiles. Scale bars $=5 \mu \mathrm{m}$. 


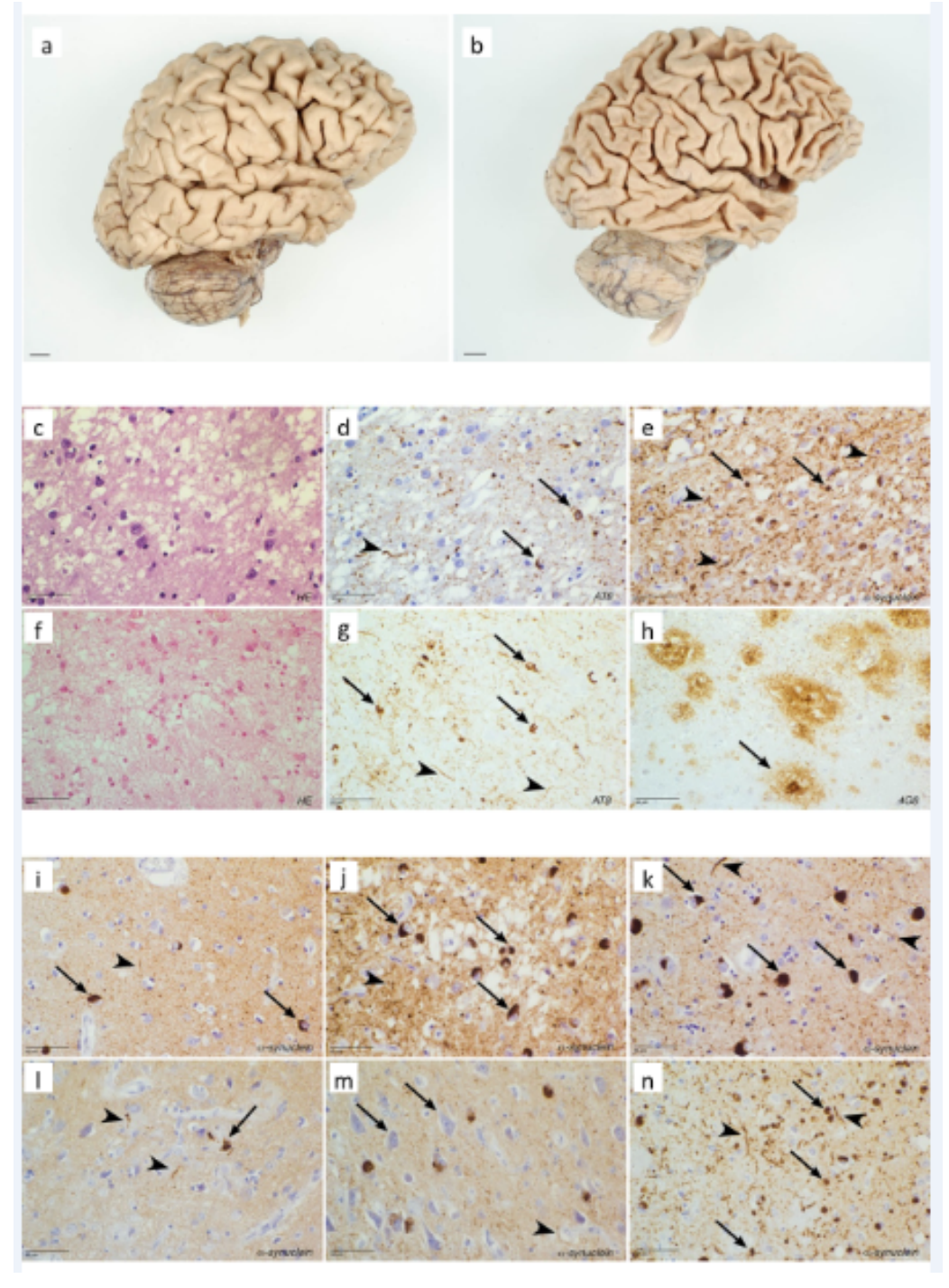

\section{Figure 4}

Neuropathology of patient carriers P1 and P3. a. Right hemisphere of patient P1 shows moderate frontotemporal atrophy, with the superior temporal gyrus more affected than the medial temporal gyrus. b. Right hemisphere of patient P3 shows severe cortical atrophy, more pronounced in the frontal and temporal lobes. The pre- and post-central gyrus are relatively spared. c-e. The lateral occipitotemporal gyrus of patient P1 shows (c) microspongiotic changes in the cortex (Hematoxylin-Eosin stain), (d) a relatively mild load of neurofibrillary tangles (arrow) and neuritic threads (arrowhead) (AT8 stain), and (e) severe a-synuclein pathology with Lewy bodies (arrow) and Lewy neurites (arrow head). f-h. The frontal cortex of patient P3 shows ( $f$ ) severe microspongiosis and neuronal loss (Hematoxylin-Eosin stain), (g) the abundance of hyperphosphorylated tau pathology with many neurofibrillary tangles (arrow) and neuritic threads (arrowhead) (AT8 stain), and (h) severe $\beta$-amyloid pathology with many classic (arrow) and diffuse senile plaques (4G8 stain). i-k. a-synuclein pathology of patient P1 in (i) the frontal cortex, (j) the hippocampus (severe) and (k) the amygdala (severe). I-n. a-synuclein pathology of patient P3 in (I) the frontal cortex, (m) the hippocampus (moderate) and (n) the amygdala (severe). Lewy bodies are marked with arrows, Lewy neurites with arrowheads.

\section{Supplementary Files}

This is a list of supplementary files associated with this preprint. Click to download. 
- SmoldersS.etal.SupplementaryTablesandFigures.pdf 